\title{
A comparative analysis of nonhost resistance across the two Triticeae crop species wheat and barley
}

\author{
Rhoda Delventhal ${ }^{1 \dagger}$, Jeyaraman Rajaraman ${ }^{2 \dagger}$, Francesca L. Stefanato ${ }^{3,8}$, Sajid Rehman ${ }^{4,9}$, Reza Aghnoum ${ }^{4,10}$, \\ Graham R. D. McGrann ${ }^{3}$, Marie Bolger ${ }^{5}$, Björn Usadel ${ }^{5}$, Pete E. Hedley ${ }^{6}$, Lesley Boyd ${ }^{7}$, Rients E. Niks ${ }^{4}$, \\ Patrick Schweizer ${ }^{2^{*}}$ and Ulrich Schaffrath ${ }^{1 *}$ (D)
}

\begin{abstract}
Background: Nonhost resistance (NHR) protects plants against a vast number of non-adapted pathogens which implicates a potential exploitation as source for novel disease resistance strategies. Aiming at a fundamental understanding of NHR a global analysis of transcriptome reprogramming in the economically important Triticeae cereals wheat and barley, comparing host and nonhost interactions in three major fungal pathosystems responsible for powdery mildew (Blumeria graminis ff. ssp.), cereal blast (Magnaporthe sp.) and leaf rust (Puccinia sp.) diseases, was performed.

Results: In each pathosystem a significant transcriptome reprogramming by adapted- or non-adapted pathogen isolates was observed, with considerable overlap between Blumeria, Magnaporthe and Puccinia. Small subsets of these general pathogen-regulated genes were identified as differentially regulated between host and corresponding nonhost interactions, indicating a fine-tuning of the general pathogen response during the course of co-evolution. Additionally, the host- or nonhost-related responses were rather specific for each pair of adapted and non-adapted isolates, indicating that the nonhost resistance-related responses were to a great extent pathosystem-specific. This pathosystem-specific reprogramming may reflect different resistance mechanisms operating against non-adapted pathogens with different lifestyles, or equally, different co-option of the hosts by the adapted isolates to create an optimal environment for infection. To compare the transcriptional reprogramming between wheat and barley, putative orthologues were identified. Within the wheat and barley general pathogen-regulated genes, temporal expression profiles of orthologues looked similar, indicating conserved general responses in Triticeae against fungal attack. However, the comparison of orthologues differentially expressed between host and nonhost interactions revealed fewer commonalities between wheat and barley, but rather suggested different host or nonhost responses in the two cereal species.
\end{abstract}

Conclusions: Taken together, our results suggest independent co-evolutionary forces acting on host pathosystems mirrored by barley- or wheat-specific nonhost responses. As a result of evolutionary processes, at least for the pathosystems investigated, NHR appears to rely on rather specific plant responses.

Keywords: Wheat, Barley, Blumeria, Magnaporthe, Puccinia, Adapted isolate, Non-adapted isolate, Nonhost resistance, Quantitative resistance, Global transcriptome analysis

\footnotetext{
*Correspondence: schweiz@ipk-gatersleben.de; schaffrath@bio3.rwth-

aachen.de

${ }^{\dagger}$ Equal contributors

'Leibniz-Institute of Plant Genetics and Crop Plant Research, 06466

Gatersleben, Germany

'Department of Plant Physiology, RWTH Aachen University, 52056 Aachen,

Germany

Full list of author information is available at the end of the article
} 


\section{Background}

The Triticeae include some of our most important cereal crops, with wheat (Triticum aestivum) and barley (Hordeum vulgare) accounting for almost one third of the world's cereal production $[1,2]$. With pests and diseases being responsible for $28 \%$ of wheat losses, resistance breeding is of great importance for food security [3-5]. Disease resistance may rely on preformed or inducible defense mechanisms, the latter requiring plant recognition of conserved pathogen-associated molecular patterns (PAMPs) and/or specific effectors that the pathogen has evolved to suppress plant defense or induce susceptibility $[6,7]$. In host interactions basal resistance is regarded as part of the PAMP-Triggered Immunity (PTI) response, and if not suppressed by effectors can remain effective against an adapted pathogen as a quantitative, but relatively stable resistance [7, 8]. In contrast, Effector-Triggered Immunity (ETI) is a qualitative resistance, usually based on single major resistance $(R-)$ genes acting upon effector, in this case termed avirulence factor, recognition. Although conferring complete immunity, $R$-gene resistance can be broken relatively easily by pathogen variants expressing mutated target effectors (virulence factor) [9].

Nonhost resistance (NHR), which by definition protects all individuals (genotypes) of a plant species against all isolates of a would-be pathogen, is seen as a novel source of durable and broad spectrum resistance [10-12]. In most interactions between plants and non-adapted pathogens, resistance has been shown to rely upon several genes that quantitatively contribute to NHR, e.g. NHR in barley to the wheat leaf rust (Puccinia triticina; Pt) and the wheat powdery mildew (Blumeria graminis f. sp. tritici; Bgt) pathogens [13-15]. However, cases of Mendelian NHR, conferred by a single gene, have also been reported, e.g. barley NHR to the maize pathogen Cochliobolus carbonum [16, 17].

Despite its potential impact on food security, the molecular frameworks underlying polygenic NHR in the Triticeae are poorly defined. It has been proposed that NHR might either be associated with PTI, whose mechanisms would suffice to defend against the non-adapted pathogen, or might involve stacked $R$-genes supporting each other by functional redundancy [18]. Unsolved questions in understanding NHR, which have driven the current study, include (i) the overlap of NHR with host resistance, (ii) whether the same NHR genes and mechanisms are effective against different pathogens, and (iii) whether NHR genes and mechanisms are conserved across different species within the Triticeae. The second question was to some extent addressed by Zellerhoff et al. [19] in a study comparing the transcriptional nonhost responses of barley to non-adapted isolates of the fungal pathogens Blumeria sp., Puccinia sp. and Magnaporthe sp. The results of that study suggested that defense responses are in part pathosystem specific. However, to the best of our knowledge no direct comparison of NHR between species of the Triticeae has been reported.

A crucial aspect in studies of NHR is the choice of the plant-pathogen interactions, in particular with respect to the relationship between the nonhost and host plant species, and between the non-adapted and adapted pathogen isolates, as evolutionary proximity often is supposed to correlate with a larger overlap between host and nonhost defense responses [20]. For wheat and barley, pathosystems are available where adapted and non-adapted isolates are closely related, allowing a within pathosystem comparison of host and nonhost interactions, and a comparison of host and nonhost interactions between wheat and barley. Such pathosystems include the causal agents of the economically important diseases of powdery mildew (Blumeria graminis), cereal blast (Magnaporthe sp.) and cereal rust (Puccinia sp.). While rusts and powdery mildews have been important field diseases of both wheat and barley since domestication of these cereal crops, wheat blast has emerged as a field disease only since 1985, and is now present in South America and Asia [21-23].

These three pathosystems have distinct lifestyles, making them interesting subjects for a study of NHR. The obligate biotroph Blumeria only invades epidermal tissue, producing feeding structures, haustoria, within epidermal cells, and subsequently grows as an ectoparasite on the plant surface [24]. Puccinia, also an obligate biotroph, enters the plant through stomata, producing haustoria within mesophyll cells and grows mainly intercellularly [25]. In contrast, Magnaporthe isolates have a hemi-biotrophic lifestyle. They penetrate the plant epidermis forming invasive 'haustoria-like' hyphae in the initial biotrophic phase, then enter a necrotrophic phase, destroying the colonized epidermal and mesophyll tissue $[26,27]$. Despite the different lifestyles, in all three pathosystems the first $48 \mathrm{~h}$ after spore germination are crucial for the outcome of the infection attempts. During this period the adapted isolates successfully invade the plant, while the growth of the nonadapted isolates is arrested, with plant defense responses occurring during or shortly after penetration [28-30].

In this study we investigate the early responses of wheat and barley to adapted and non-adapted isolates of the pathosystems Blumeria sp., Magnaporthe sp. and Puccinia $s p$. The study focused on the initial tissue colonization phase which is most crucial for initiation of effective defense responses. The later stages of host-colonization, in particular the reproductive stages, were excluded as the non-adapted isolates never reach these developmental stages. The objectives of this study were (i) to identify specific nonhost responses from general pathogen-regulated plant responses, (ii) to examine commonalities in nonhost responses between the three pathosystems and (iii) to compare nonhost-related responses between wheat and barley. 
Importantly, our results point to a high level of specificity of nonhost responses, both for pathosystems and for plant species.

\section{Results}

A major goal of our study was the examination of transcriptional reprogramming in wheat and barley towards three fungal pathosystems (Blumeria, Magnaporthe and Puccinia), comparing responses to adapted (host interactions) and non-adapted (nonhost interaction) isolates. To enable comparability we selected adapted isolates that displayed virulence in the host interaction and non-adapted isolates that evoked a rapid, cell-autonomous immune response in the nonhost situation on both wheat and barley. All experiments were conducted on the wheat cultivar Renan and the barley cultivar Vada.

\section{Infection phenotypes on wheat and barley in host and nonhost interactions}

The wheat and barley isolates causing powdery mildew (Bgt and $B$. graminis f. sp. hordei, Bgh) and the isolates causing leaf rust ( $P t$, and $P$. hordei, $P h$ ) were fully virulent on their respective host plant species, but showed no macroscopic symptoms on the nonhost species (Fig. 1a). This absence of lesions was previously defined as type I NHR [6]. The Magnaporthe pathosystem (anamorph: Pyricularia) was represented by the adapted M. oryzae (Mo) isolates Br116.5 and TH6772, which caused a similar disease phenotype on wheat and barley, respectively (Fig. 1a). An isolate of the closely related Magnaporthe species, Pyricularia penniseticola (Pp, CD180) [31], isolated from Pennisetum sp., was used as the non-adapted Magnaporthe isolate for both wheat and barley, and produced no visible disease symptoms on either cereal species (Fig. 1a).

To identify at which stage in development (Fig. 1b) the non-adapted isolate ceased growth in the nonhost interaction, all interactions were investigated by microscopy at selected time points after inoculation (Additional file 1: Figure S1, Additional file 2: Figure S2, Additional file 3: Figure S3). In general, spores of all the fungal isolates were able to form germ tubes and appressoria on the leaf surface of both host and nonhost plants. This indicated that NHR did not operate at the pre-penetration stage, but rather became effective during penetration or post-penetration. While Bgh and Bgt had formed elongating secondary hyphae at $74 \mathrm{~h}$ past inoculation (hpi) in the host interactions, this developmental stage was never observed in the respective nonhost interactions (Additional file 1: Figure S1). Early plant responses included the accumulation of autofluorescent material underneath the appressorium or autofluoresence of the whole attacked epidermal cell, indicative of papilla-associated defense and initiation of hypersensitive response (HR), respectively [32, 33]. These reactions were observed at the majority of Bgt interaction sites, on wheat and barley, at $12 \mathrm{hpi}$, while in response to Bgh papillae or HR responses were first seen at $24 \mathrm{hpi}$ (Additional file 1: Figure S1). This could indicate that $B g t$ is generally perceived faster than $B g h$, and thus evokes an earlier plant reaction (irrespective of the host-status of the plant). However, the levels of epidermal HR were higher in the nonhost than in the host interactions at $24 \mathrm{hpi}$ (Additional file 1: Figure S1) indicating a specific role of this defense response in NHR against Blumeria.

The blast isolates, $M o$ and $P p$, had formed appressoria by $6-12$ hpi in both the host and nonhost interactions (Additional file 2: Figure S2). The adapted $M o$ isolates Br116.5 and TH6772 were able to penetrate the epidermis of wheat and barley, respectively, invasive growth being first seen at $24 \mathrm{hpi}$. In contrast, the non-adapted $P p$ isolate CD180 was mostly arrested after appressorium formation (Additional file 2: Figure S2). While on wheat penetration attempts by CD180 failed completely, on barley invasive growth of CD180 was seen at a few interaction sites. Accordingly, the only autofluorescence response in wheat was seen associated with papilla formation, whereas barley responded to the non-adapted isolate with papillae as well as HR (Additional file 2: Figure S2). This implied that in wheat, at least in cv. Renan, penetration barriers might have been sufficient to arrest growth of CD180, while in the barley cv. Vada NHR operated at the penetration stage as well as post-penetration.

The rust fungi $P t$ and $P h$ entered through stomata and formed haustorial mother cells (HMC) in both host and nonhost interactions within 24 hpi (Additional file 3: Figure S3). $P h$ developed more rapidly than $P t$, and had formed more infection hyphae and HMC at 12 hpi than $P t$ on both wheat and barley. Differences between host and nonhost interactions were first seen at $48 \mathrm{hpi}$, when the adapted isolates had formed haustoria and branching hyphae, while the non-adapted isolates were not able to colonize the mesophyll (Additional file 3: Figure S3).

\section{Reprogramming of the wheat and barley transcriptome in response to pathogen inoculation}

Transcriptional analyses were performed on samples of inoculated and non- or mock-inoculated leaf tissues collected at four critical time points in the different pathosystems (Fig. 1b). As the entire Blumeria and the critical, early Magnaporthe interactions were confined to the cereal epidermis (Fig. 1b), tissue samples were taken only of the abaxial epidermis to optimize the concentration of transcripts impacted by the interactions. Puccinia interactions involved multiple leaf cell types (Fig. 1b), therefore whole leaves were sampled.

To identify genes differentially expressed in response to pathogen inoculation (both adapted or non-adapted isolates), inoculated and mock-inoculated samples were compared using the same statistical workflow for each 


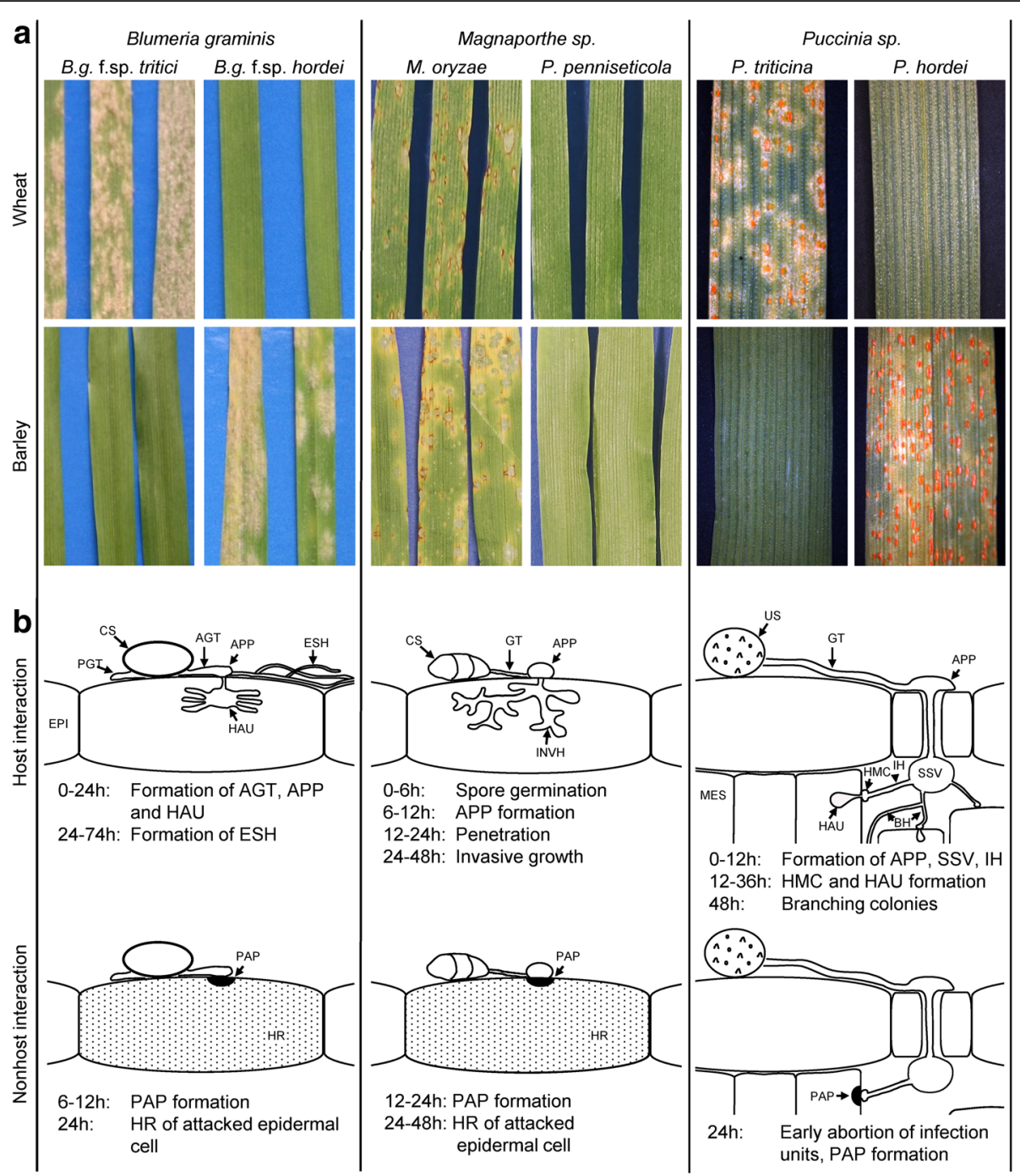

Fig. 1 Phenotypes of host and nonhost interactions of wheat and barley with Blumeria, Magnaporthe and Puccinia. a, Macroscopic phenotypes of wheat cv. Renan and barley cv. Vada 10 days after inoculation with Blumeria isolates, 7 days after inoculation with Magnaporthe isolates and 20 days after inoculation with Puccinia isolates. b. Cytological phenotypes of host and nonhost interactions (summarized and simplified from Additional files 1, 2 and 3). Upper charts show typical fungal infection structures and their time of appearance as observed in host interactions. Lower charts show developmental stages in which fungal growth is typically arrested (and plant responses involved) in respective nonhost interactions. AGT: appressorial germ tube, APP: appressorium, BH: branching hyphae, CS: conidiospore, EPI: epidermis, ESH: elongating secondary hyphae, GT: germ tube, HAU: haustorium, HMC: haustorial mother cell, HR: hypersensitive response, IH: infection hypha, INVH: invasive hyphae, MES: mesophyll, PAP: papilla, PGT: primary germ tube, US: urediniospore, SSV: substomatal vesicle

pathosystem (Additional file 4: Table S1). These differentially expressed genes (DEGs) were subsequently referred to as the general pathogen-regulated genes. Overall, both wheat and barley reacted to pathogen inoculation with significant changes in transcript abundance of a great number of genes. In all pathosystems, except for barleyBlumeria, more DEGs were on average up-regulated than down-regulated after inoculation (Additional file 5: Figure S4). A considerable number of genes were regulated in common between the three pathosystems, in both wheat and barley, suggesting the existence of a biotic stress-related core transcriptome in the two cereals.
In wheat, the number of DEGs was higher in the Puccinia pathosystem (10,756 DEGs) than in the Blumeria (4811 DEGs) or the Magnaporthe (2777 DEGs) pathosystems (Fig. 2a). Overall the combined number of wheat genes regulated in response to at least one of three pathogens was 12,215 , which was taken as base for subsequent calculations. A high percentage of wheat DEGs (31\%) were in common between the Blumeria and Puccinia pathosystem (Fig. 2a, orange and grey intersections), while 14 and $18 \%$ were in common between Blumeria and Magnaporthe (purple and grey), and Magnaporthe and Puccinia (green and grey), respectively (Fig. 2a). In wheat $13 \%$ of DEGs 


\section{a}

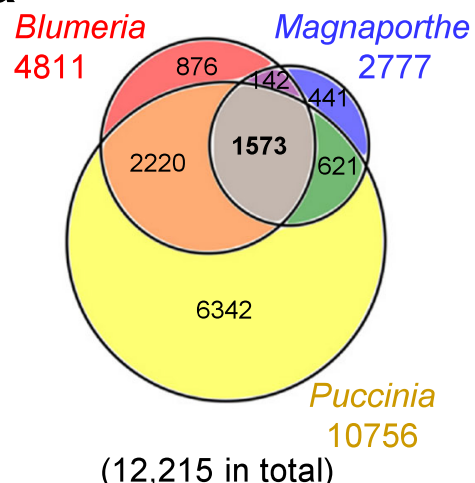

b

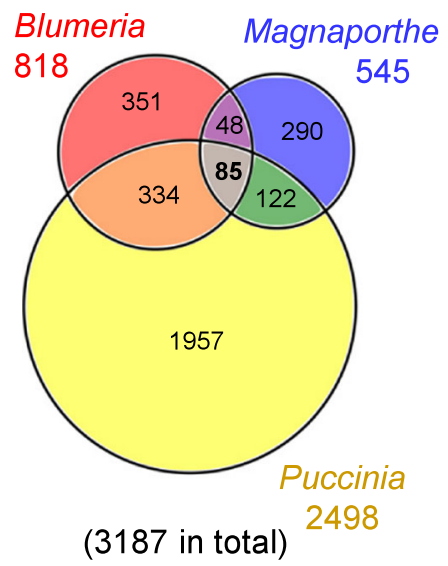

Barley

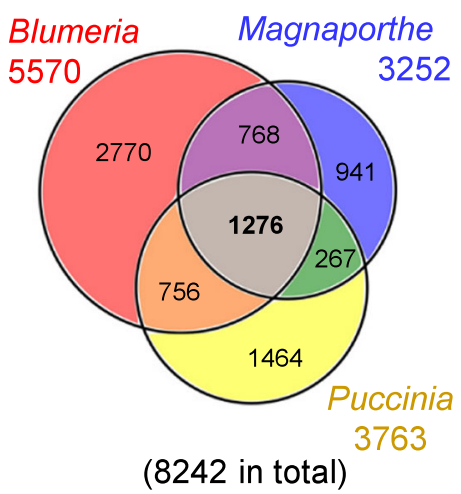

Blumeria

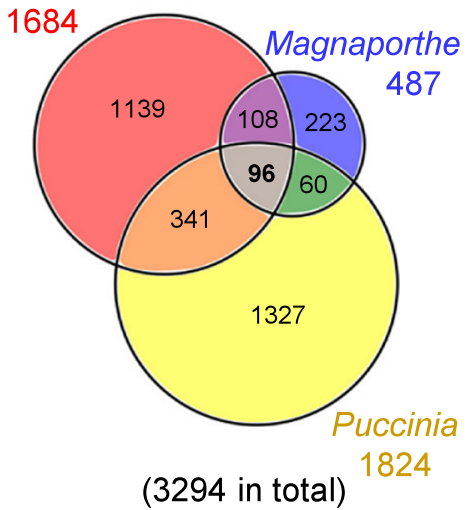

Fig. 2 Identification of differentially expressed genes in wheat and barley for three pathosystems. a, The number of genes differentially expressed in wheat and barley for the three pathosystems Blumeria, Magnaporthe and Puccinia comparing inoculated samples (both adapted and non-adapted isolates) to mock inoculation. $\mathbf{b}$, Those genes identified in (a) differentially expressed in wheat and barley for the three pathosystems Blumeria, Magnaporthe and Puccinia comparing samples inoculated with the adapted versus non-adapted isolates

were shared between all three pathosystems (Fig. 2a, grey intersection). In barley, compared to wheat, similar numbers of DEGs were identified in the Blumeria (5570 DEGs) and the Magnaporthe (3252 DEGs) pathosystems, whereas fewer DEGs (3763) were found for the Puccinia pathosystem (Fig. 2a). Comparing the pathosystems, 25, 25 and 19\% of the barley DEGs were shared between Blumeria and Puccinia (Fig. 2a, orange and grey), Blumeria and Magnaporthe (purple and grey), and Magnaporthe and Puccinia (green and grey), respectively (Fig. 2a). In barley, similar to wheat, $15 \%$ of DEGs were shared between all three pathosystems (Fig. 2a, grey intersection).

\section{Differential reprogramming of the wheat and barley transcriptome during host and nonhost interactions}

To examine the differences between host and nonhost interactions in each pathosystem, DEGs were identified within the sets of general pathogen-regulated genes established above, by comparing samples from inoculations with adapted isolates to samples inoculated with nonadapted isolates (Additional file 6: Table S2). The number of genes differentially expressed between host and nonhost interactions were found to be less than $20 \%$ of the general pathogen-regulated genes for the pathosystems wheat-Blumeria, wheat-Magnaporthe and barley-Magnaporthe, and less than $50 \%$ for the other pathosystems (Fig. 2a and b). This indicated that the differential response of the plant transcriptome to adapted versus nonadapted isolates was relatively small, and that the general pathogen-induced reprogramming occurred rather irrespective of the isolate being adapted or non-adapted.

In both wheat and barley, the largest number of nonhostrelated DEGs were identified in the Puccinia pathosystem, whereas the Magnaporthe pathosystem had the fewest nonhost-related DEGs (Fig. 2b). While 13\% of DEGs were in common between the Blumeria and Puccinia pathosystems both in wheat and barley (Fig. 2b, orange and grey intersections), the other pathosystem comparisons revealed 
only 4-6\% of shared DEGs (Fig. 2b). In both wheat and barley, the three pathosystems Blumeria, Magnaporthe and Puccinia had only $3 \%$ of the nonhost-related DEGs in common (Fig. 2b, grey intersections, Additional file 6: Table S2). Altogether, the nonhost-related DEGs showed only small overlaps between the different cerealpathosystems (Fig. 2b) compared to the general pathogenregulated genes (Fig. 2a).

\section{Conserved pathogen-induced transcriptional reprogramming between wheat and barley}

To more directly interrogate the conservation of pathogen-induced transcriptional reprogramming between wheat and barley, putative gene orthologues had to be first identified. Two approaches were undertaken to identify the orthologues: (i) identification of reciprocal best blast hits in unigene sets of wheat and barley received from TaGI and HarvEST databases, respectively, and (ii) identification of orthologue clusters within the TaGI wheat unigenes and the IBSC barley coding sequences using the InParanoid tool [34] (Additional file 7: Table S3). In summary, 57.8 and $38.0 \%$ of the wheat and barley microarray probes could be linked by orthologue matching (Additional file 7: Table S3). We observed redundant linking of $37 \%$ of orthologue-assigned barley genes to more than one wheat orthologue, whereas only $7.1 \%$ of the orthologue-assigned wheat genes were assigned to more than one barley orthologue. In part, this redundancy might be attributed to the allohexaploid status of wheat, which would assign every barley gene to up to three wheat homoeologues.

To identify similarities in the transcriptional reprogramming in wheat and barley to pathogen inoculation the general pathogen-regulated DEGs were screened for matching orthologues (Fig. 3a). Out of the 4811 wheat DEGs found for the Blumeria pathosystem (Fig. 3a, whole bar) a total of 3152 DEGs had at least one orthologue in barley (Fig. 3a, grey and black sections), of which 1306 wheat DEGs had orthologues also present in the list of barley-Blumeria DEGs (Fig. 3a, black section). Conversely, of 5570 Blumeria-regulated barley DEGs, 2322 had orthologues in wheat, of which 946 had orthologues that were differentially regulated in the wheat-Blumeria pathosystem (Fig. 3a). In conclusion, $41 \%$ of the orthologue-assigned DEGs were general pathogen-regulated following Blumeria inoculation, in both wheat and barley. Similar percentages were found for the Magnaporthe pathosystem: $41 \%$ of the orthologueassigned wheat DEGs and 39\% of the orthologue-assigned

\section{a}

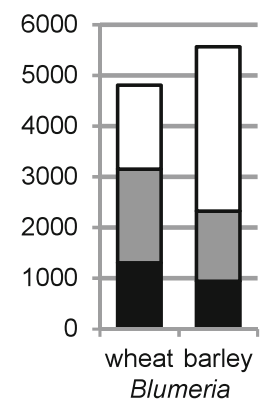

General pathogen-regulated genes
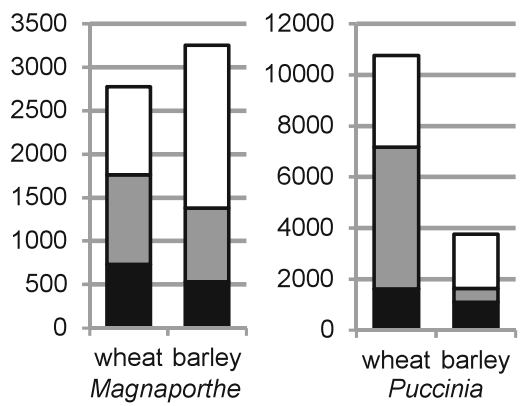

b

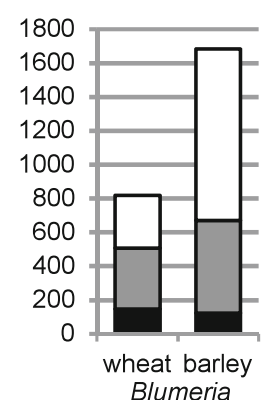

Host versus nonhost-regulated genes
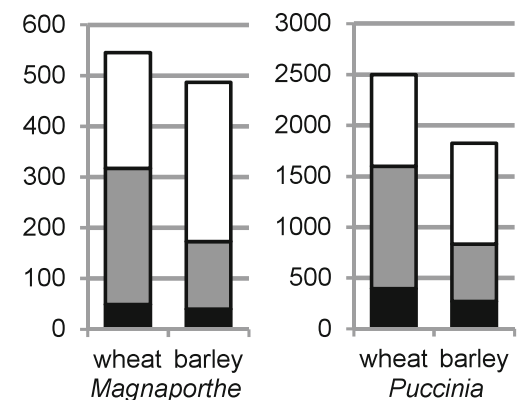

$\square$ No

orthologues

At least one

orthologue

in other

cereal, but

not DEG

At least one

orthologue

and DEG in

both cereal

species

Fig. 3 Comparison of differentially expressed genes identified in wheat and barley. Orthologous genes identified between wheat and barley were compared for each pathosystem, in both $\mathbf{a}$, the general pathogen-regulated genes, and $\mathbf{b}$, the host versus nonhost regulated genes. The total number of each gene list is shown as whole bars. For each list, the portion of genes that could be assigned to a putative orthologue is highlighted (grey and black) and comprises a subset whose orthologues were also present in the according gene list of the other cereal species (black). The black sections are represented in heat maps (Figs. 4, 5, 6 and 7) 
barley DEGs had Magnaporthe-regulated orthologues in barley and wheat, respectively. In the Puccinia pathosystem, $23 \%$ of the orthologue-assigned wheat DEGs and 68\% of the orthologue-assigned barley DEGs had Puccinia-regulated orthologues in the other cereal species. Taking into account that the number of DEGs found for the wheatPuccinia interaction exceeded the number of barley-Puccinia-DEGs threefold (Fig. 2a), these percentages can be considered similar to those found for the other pathosystems. In each case the number of general pathogen regulated DEGs in the overlap of wheat and barley was larger than expected by chance (Additional file 8: Data S1). Taken together, the results indicated, that there exists a conserved general pathogen-regulated transcriptional response in wheat and barley.

To examine the expression profiles of the orthologous general pathogen-regulated DEGs in wheat and barley over time heat maps were constructed (Figs. 4, 5, 6 and Additional file 9: Table S4). The wheat DEGs, for which orthologous barley DEGs had been identified, were clustered hierarchically according to their expression profiles in the Blumeria, Magnaporthe and Puccinia interactions, and the expression profiles of the corresponding barley orthologues placed alongside (Figs. 4, 5 and 6). In general, the wheat heat maps were very similar to the according barley heat maps, confirming that wheat and barley share a common pathogen-induced transcriptional response.

\section{Commonalities of the conserved transcriptional reprogramming in response to pathogens with different life-styles}

To learn more about the function of the general pathogen-regulated gene orthologues, wheat and barley transcripts were assigned to functional categories using MapMan [35, 36]. Each gene cluster presented in Figs. 4, 5 and 6 was subjected to an over-representation analysis (ORA), pointing to those functional categories over- or under-represented within the gene set.

With the Blumeria pathosystem (Fig. 4), hierarchical clustering of the wheat genes and assignment of their barley orthologues resulted in clusters $\mathrm{B}, \mathrm{C}$ and $\mathrm{D}$ being downregulated, while clusters $\mathrm{F}, \mathrm{G}$ and $\mathrm{H}$ were up-regulated after inoculation with adapted and non-adapted isolates (Fig. 4). The MapMan categories 'secondary metabolism,' 'hormone metabolism' and 'stress' were significantly over-represented in several of these clusters. The category 'cell wall' was over-represented in the down-regulated clusters of genes (Fig. 4, clusters B, C, D), suggesting that down-regulation of normal cell wall metabolism is a common response in both wheat and barley to adapted and non-adapted Blumeria isolates, possibly allowing the formation of de-novo cell wall components by pathogen-induced gene products. The high importance for cell-wall re-construction for resistance to
Blumeria has been shown [37-39]. The up-regulation of DEGs in cluster F clearly peaked at $24 \mathrm{hpi}$, in both host and nonhost interactions, in wheat and in barley (Fig. 4). In cluster $\mathrm{F}$ the functional categories 'stress,' 'secondary metabolism,' 'amino acid metabolism' and 'miscellaneous enzyme families' (including glucan endo-1,3-beta-glucosidases, cytochrome P450s, glutathione-S-transferases and peroxidases) were significantly over-represented. The results for 'secondary metabolism,' 'amino acid metabolism' are in agreement with and extend reports on strong activation of the shikimate and phenylpropanoid pathways in Blumeriaattacked barley and wheat [40, 41]. In clusters $G$ and $H$ most wheat DEGs showed an earlier up-regulation in response to adapted and non-adapted Blumeria isolates (peaking at 6 or $12 \mathrm{hpi}$ ) than their corresponding barley orthologues (peaking at 12 or $24 \mathrm{hpi}$ ). In these clusters the category 'protein' was significantly under-represented, while 'stress', miscellaneous enzyme families,' signaling' and 'transport' were significantly over-represented (Fig. 4). This would be in agreement with reports on signaling and transport events being initiated during the early plant-pathogen infection stages [42]. Overall, despite the timing difference between wheat and barley mentioned for clusters $G$ and $H$, the transcriptional regulation of the orthologues was highly conserved.

The functions of wheat and barley orthologous DEGs in the Magnaporthe pathosystem in many respects resembled those in the Blumeria pathosystem (Fig. 5). The transcription profiles in wheat and in barley looked very similar in adapted and non-adapted isolate interactions. Within the down-regulated wheat and barley DEG orthologues, the MapMan categories 'lipid metabolism,' 'stress', 'secondary metabolism' and 'cell wall' were over-represented (Fig. 5, cluster A). As with the Blumeria pathosystem most upregulated wheat DEGs responded earlier in response to Magnaporthe isolates than their corresponding barley orthologues (Fig. 5, cluster C). However, because only one cultivar per plant species was used, it remains unclear to what extent this difference reflects a species- or cultivarspecific effect. The corresponding DEGs showed a significant over-representation in categories 'transport' and 'signaling'. The wheat genes up-regulated at $24 \mathrm{hpi}$ in general had barley orthologues also up-regulated at $24 \mathrm{hpi}$, and showed over-representation in 'amino acid metabolism, 'secondary metabolism,' 'miscellaneous enzyme families' and 'stress' (Fig. 5, cluster D). In both the Blumeria and Magnaporthe interactions at 24 hpi papillae and hypersensitive cell responses were observed at high frequencies (Additional file 1: Figure S1, Additional file 2: Figure S2), suggesting that regulation of these DEGs may be associated with these plant defense responses.

In the case of the Puccinia pathosystem, the expression profiles of the wheat DEGs resembled those of their barley orthologues with regard to up- or down-regulation, but 


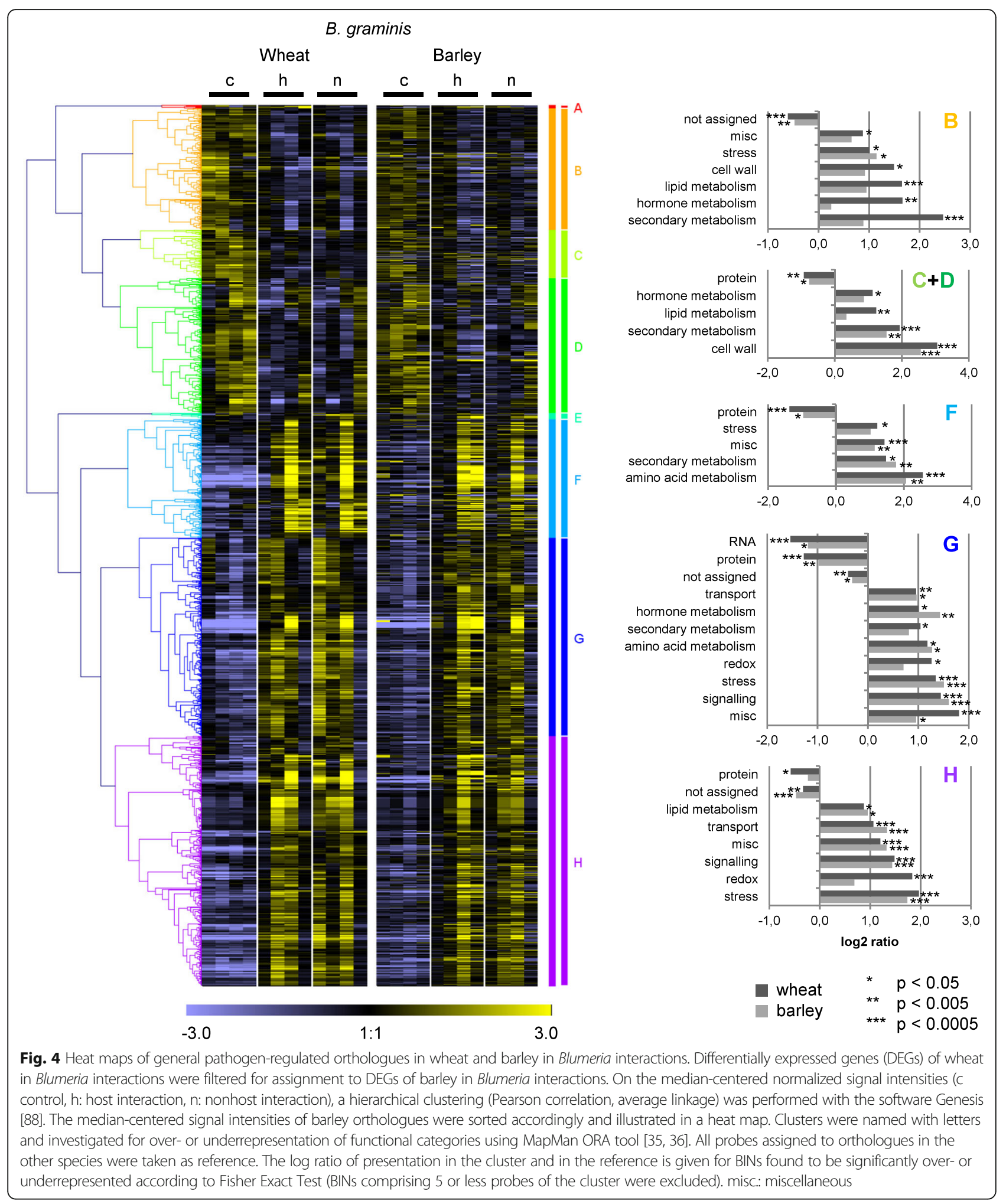




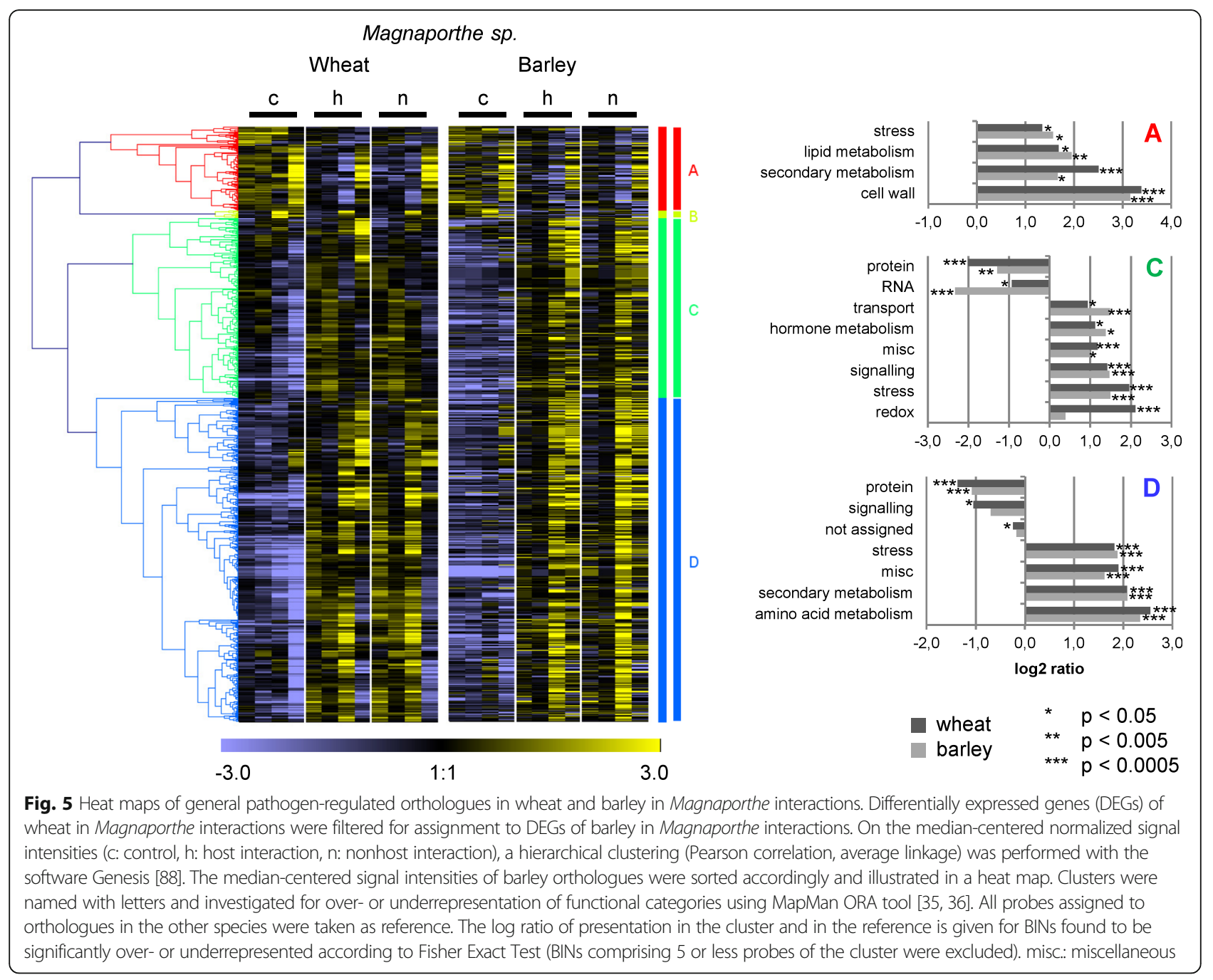

often differed with respect to expression kinetics (Fig. 6). Wheat DEGs, that were very early and similarly upregulated after inoculation with adapted and non-adapted isolates, had barley orthologues showing a delayed response which was more pronounced in the host than in the nonhost interaction (Fig. 6, cluster D). In part, this might be due to the faster development of $P h$ in comparison to $P t$, as indicated by the microscopy (Additional file 3: Figure S3). Over all clusters, both wheat and barley showed more pronounced gene regulation in response to the $P h$ than to the $P t$ isolate, irrespective of the host or nonhost status of the plant, which indicates again a major effect of the Puccinia species per se (Fig. 6). In contrast to Blumeria and Magnaporthe, photosynthesis-associated genes were overrepresented within the clusters of down-regulated genes after Puccinia inoculation (Fig. 6, clusters A and C), probably because whole leaf tissue was sampled to study Puccinia interactions, whereas epidermal tissue was sampled for Blumeria and Magnaporthe. However, the transcriptional reprogramming after Puccinia inoculation also shared obvious commonalities with the Blumeria and Magnaporthe pathosystems: Within the down-regulated DEGs the functional categories 'cell wall' and 'lipid metabolism' were significantly over-represented (Fig. 6, clusters A and C), while up-regulated DEGs were often associated with 'secondary metabolism,' 'stress', 'signaling,' 'miscellaneous enzymes', 'transport' and 'amino acid metabolism' (Fig. 6, clusters D, F and G), in agreement with major Puccinia-regulated functional categories determined by RNA sequencing [43].

In summary, a future more detailed analysis of the genes in the most strongly over-represented MapMan bins (with $\log 2$ ratio of more than 2 in both species) such as "cell wall" in the Blumeria and Magnaporthe interactions, "OPP" in the Puccinia interaction, and "secondary metabolism" plus "amino acid metabolism" in all pathosystems might guide us towards important genes involved in the plant responses to one specific or several pathosystems. 


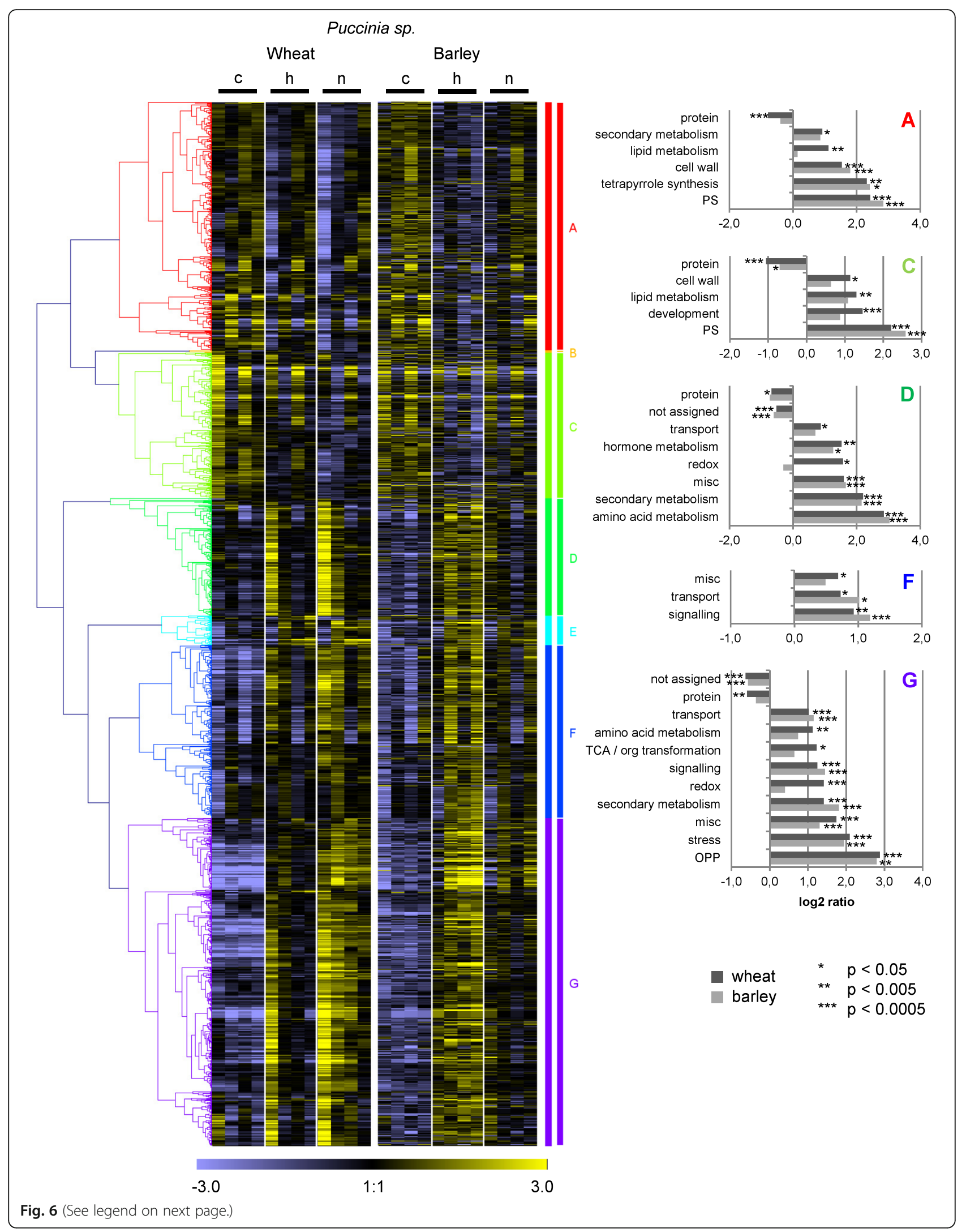


(See figure on previous page.)

Fig. 6 Heat maps of generally pathogen-regulated orthologues in wheat and barley in Puccinia interactions. Differentially expressed genes (DEGs) of wheat in Puccinia interactions were filtered for assignment to DEGs of barley in Puccinia interactions. On the median-centered normalized signal intensities (c: control, h: host interaction, n: nonhost interaction), a hierarchical clustering (Pearson correlation, average linkage) was performed with the software Genesis [88]. The median-centered signal intensities of barley orthologues were sorted accordingly and illustrated in a heat map. Clusters were named with letters and investigated for over- or underrepresentation of functional categories using MapMan ORA tool $[35,36]$. All probes assigned to orthologues in the other species were taken as reference. The log ratio of presentation in the cluster and in the reference is given for BINs found to be significantly over- or

underrepresented according to Fisher Exact Test (BINs comprising 5 or less probes of the cluster were excluded). PS, photosynthesis; misc:. miscellaneous

\section{Differences in nonhost-specific transcriptional reprogramming between wheat and barley}

To examine whether wheat and barley share a nonhostrelated transcriptional response, in all or any of the pathosystems studied, the nonhost-related DEGs were also searched for matching orthologues (Fig. 3b). Overall, the percentages of DEGs that were assigned an orthologous DEG in the other species were lower than found for the general pathogen-regulated DEGs (Fig. 3b). For the Blumeria pathosystem 29\% of the orthologue-assigned wheat DEGs and 19\% of the orthologue-assigned barley DEGs had Blumeria-regulated orthologues in barley and wheat, respectively (Fig. 3b). For the Magnaporthe pathosystem the corresponding percentages were 15 and 23\%, while for the Puccinia pathosystem they were 25 and 32\% (Fig. 3b). As for the general pathogen-regulated DEGs, the expression profiles of the nonhost-related wheat DEGs were analyzed by hierarchical clustering, the results being depicted alongside the respective barley orthologues in heat maps (Fig. 7, Additional file 9: Table S4).

With the Blumeria pathosystem many wheat DEGs showed higher expression in the nonhost interaction compared to the host interaction at $6 \mathrm{hpi}$, while this very early response was almost absent in barley (Fig. 7, Additional file 10: Figure S5). Instead, many orthologous barley DEGs showed a higher expression in the nonhost compared to the host interaction at $12 \mathrm{hpi}$, where most of the respective wheat orthologues were expressed at a lower level in the nonhost interaction, but re-induced at 24 hpi (Fig. 7). A number of DEG orthologues were significantly higher expressed in nonhost compared to corresponding host interactions in both cereal species, but their expression profiles showed different temporal patterns in wheat and barley (Fig. 7, red marks). At the later time points more barley than wheat DEGs showed a specific up-regulation after inoculation with the adapted Blumeria isolate (Fig. 7, Additional file 10: Figure S5), suggesting more intense stress of co-option of barley as a susceptible host. Again, it remains open to what extent this reflects a species- or cultivar-specific effect.

With the Magnaporthe pathosystem the orthologous nonhost-related DEGs were mostly up-regulated in the later stages of interactions, in particular in the host interactions, this response being more pronounced in barley than in wheat (Fig. 7). Only a few DEGs showed a higher expression in the nonhost than in the respective host interaction, in both wheat and barley, and none of them were shared by the two cereal species.

The regulation of the orthologous nonhost-related DEGs in the Puccinia interactions was generally more pronounced after inoculation with $P h$ than after inoculation with $P t$, in both wheat and barley (Fig. 7), corresponding with the faster development of $\mathrm{Ph}$ seen in the microscopic study (Additional file 3: Figure S3). Consequently, in wheat most of the nonhost-related DEGs were expressed at higher levels in the nonhost compared to the host interaction, while in barley the opposite was the case (Fig. 7). Only five wheat DEGs and their corresponding three barley orthologues showed a significantly enhanced expression in the nonhost compared to the host interaction, in both wheat and barley (at 36 or 48 hpi, Fig. 7, red marks). The corresponding unigenes (TC407329, TC398344, TC445077, TC410428, TC398384 for wheat, U35_260, U35_16207, U35_16207, U35_16207 and U35_6409 for barley) were annotated as 'WRKY transcription factor 21', 'stress-induced hydrophobic peptide' and 'Glucosyl transferase, putative'. However, their expression profiles looked rather different in wheat and barley (Fig. 7).

Taken together these results suggest that there is no significant, common nonhost-related transcriptional response in wheat and barley across pathosystems. To address the possibility that the lack of transcriptional commonalities was a consequence of a too strict orthologue-based approach, an additional, regulonbased approach was used to search for commonalities between wheat and barley nonhost-related DEGs. For each pathosystem all wheat and barley DEGs (Fig. 2b) were pooled and assigned to groups of co-regulated genes by $k$-means and subsequent hierarchical clustering of $k$-means cluster members (Additional file 11: Figure S6). For the pathosystems Blumeria, Magnaporthe and Puccinia 14, 6 and 8 groups of coregulated DEGs, which included both wheat and barley DEGs, were identified, respectively, suggesting a conserved co-regulation of several genes in both species (Additional file 11: Figure S6). These groups were subjected to functional MapMan categorization and a BlastN analysis to identify shared gene families within the trans-species meta-regulons (Additional file 12: 


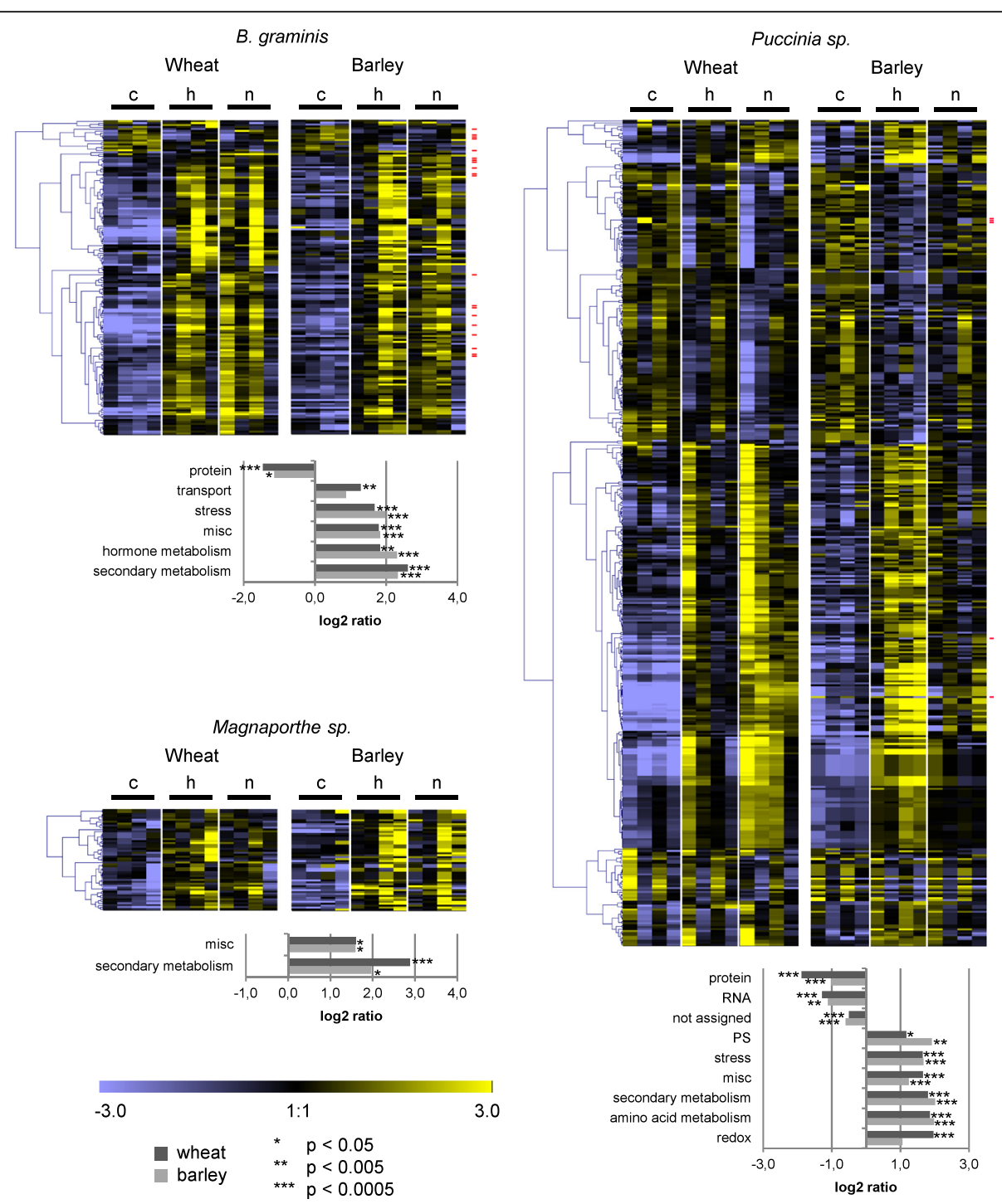

Fig. 7 Heat maps of orthologues differentially regulated between host and nonhost interactions in wheat and barley. For Blumeria, Magnaporthe and Puccinia interactions, wheat probes with differential expression between host and nonhost interaction were filtered for assignment to barley orthologues differentially expressed in the same pathosystem. On the median-centered normalized signal intensities (c: control, h: host interaction, n: nonhost interaction), a hierarchical clustering (Pearson correlation, average linkage) was performed with the software Genesis [88]. The median-centered signal intensities of barley orthologues were sorted accordingly and illustrated in a heat map. Wheat genes that showed significant higher expression in the nonhost compared to the host interaction at one time point, and were assigned to a barley orthologue with a significant higher expression in nonhost compared to host interaction, are marked in red. Over- or underrepresentation of functional MapMan categories and statistical significance according to Fisher Exact Test were calculated with MapMan ORA tool $[35,36]$. All probes assigned to orthologues in the other species were taken as reference. The log ratio of presentation in the gene subset and in the reference is shown for BINs found to be significant (BINs comprising 5 or less probes were excluded). PS, photosynthesis; OPP, oxidative pentose phosphate pathway; TCA/org, tricarboxylic acid cycle/organic acid transformation; misc:: miscellaneous

Table S5). However, neither similar functions nor significant sequence similarities were found comparing co-regulated wheat and barley DEGs in each pathosystem (Additional file 12: Table S5). This further supported the conclusion that nonhost-related transcriptional reprogramming in each pathosystem was to a high extent plant-species specific, not being conserved between wheat and barley.

\section{Discussion}

NHR is considered the most common form of disease resistance in plants, protecting all individuals of a plant species against all isolates of a would-be pathogen [10, 44]. Because most studies of NHR have been limited to the comparison of adapted and non-adapted isolates of a single pathosystem, they did not address the broadspectrum aspect of NHR. In the present study we take a 
wider view of NHR, comparing multiple pathosystems and two major cereal species of the Triticeae tribe of grasses. We asked the question whether similar gene sets are involved in NHR towards different Triticeae pathosystems by examining the transcriptional reprogramming in wheat and barley after inoculation with adapted and non-adapted isolates of Blumeria ff. ssp., Magnaporthe sp. and Puccinia sp. By dissecting the differential response between each host and nonhost interaction from the general pathogen-regulated response in each pathosystem, we aimed to identify those genes that might be involved specifically in NHR. We found a high degree of specificity in the nonhost-related responses between pathosystems, suggesting that different sets of genes are involved in the NHR responses operating against pathogens with different lifestyles. We also found a high degree of specificity in the nonhost-related responses of wheat and barley within a pathosystem, suggesting that independent nonhost barriers were built up against evolving pathosystems after Triticeae speciation, and during subsequent host-pathogen co-evolution.

\section{Triticeae activate a general transcriptional regulon in response to different pathogens}

With all three pathosystems wheat and barley responded with a significant general pathogen-regulated transcriptional reprogramming that was mostly irrespective of the isolate being adapted or non-adapted. In wheat and barley 13 and $15 \%$, respectively, of the general pathogenregulated genes were shared by all three pathosystems thus constituting a core pathogenesis-related transcriptome. DEGs in this group, both in wheat and barley, showed a significant over-representation of the functional categories 'secondary metabolism,' 'miscellaneous enzymes,' 'stress,' 'hormone metabolism,' 'cell wall', 'amino acid metabolism,' 'lipid metabolism,' 'signaling' and 'transport' (Additional file 13: Figure S7a, b). A significant portion of the general pathogen-regulated genes (in total $37 \%$ ) was also shared by at least two different pathosystems, in both wheat and barley, indicating a common plant response to pathogens of different fungal genera. This common response is likely to be triggered by conserved fungal elicitors, PAMPs or effectors, which often trigger similar defense pathways $[20,45,46]$.

With the Blumeria and Magnaporthe pathosystems similar numbers of general pathogen-regulated DEGs were found in wheat and in barley, suggesting a similar quantitative response. In contrast, clearly more genes were differentially regulated in wheat than in barley in response to the Puccinia isolates. This would indicate an extraordinary sensitive perception of rust attack by wheat, at least by cv. Renan, which possesses enhanced basal resistance against rust pathogens [47]. The general pathogen-regulated reprogramming can be regarded as the core plant response triggered by fungal PAMPs, as a large part of the general pathogen-regulated DEGs were assigned in the MapMan analysis to protein functions associated with PTI [45]. The majority of the general pathogen-regulated DEGs were up-regulated with the functional categories 'signaling' and 'secondary metabolism' being significantly over-represented. Many of the 'signaling'-associated DEGs encoded receptor-like kinases (RLKs), a group of genes associated with pathogen recognition receptors (PRRs) and PTI complexes [48-50]. The majority of the DEGs assigned to 'secondary metabolism' were involved in the phenylpropanoid or flavonoid metabolic pathways, which are important for plant defense, by providing precursors for lignification, salicylic acid or phytoalexin production [51]. A common feature of DEGs down-regulated after inoculation with Blumeria, Magnaporthe and Puccinia isolates was their frequent assignment to 'cell wall metabolism'. Changes in the plant cell wall's chemical composition have often been observed following pathogen attack $[39,52]$. In bean, cell wall alterations in response to Colletotrichum attack were suggested to contribute to plant defense by making the plant cell wall less susceptible to fungal cell wall-lytic enzymes [52-54]. In this study the down-regulation of a number of cellulosesynthesis-associated genes may reflect the necessity to switch from normal to a defense-state of cell-wall metabolism making the cell wall less sensitive to fungal cellulases, which plant pathogens like Puccinia, Blumeria and Magnaporthe are known to produce [55-57]. Furthermore, the regulation of 'cell wall'-associated genes may be associated with papillae formation, which in barley have been shown to contain callose, arabinoxylan and cellulose [39].

The sub-sets of general pathogen-regulated genes (in total 63\%) that were found only with one of the pathosystems, Blumeria, Magnaporthe or Puccinia, points to plant responses specific for the pathogen genus or species. These may represent part of a PTI response that is associated with the pathogen's mode of infection and/or pathogen induced changes in the plant that are associated with the specific lifestyle of the pathogen. For example, the formation of Puccinia appressoria leads to closure of the invaded stomata [25], which may in turn cause stress-associated metabolic changes in the plant, resulting in transcriptome changes that are not directly linked to plant defense. In addition, the pathosystemspecific DEGs might reflect responses triggered by conserved effectors or, in the case of Magnaporthe, also toxins $[7,58]$. Such effectors, acting irrespective of the host or nonhost status of the plant, would probably be specific to each pathosystem [20]. The existence of pathosystem-specific effector sets that may act on closely related host and nonhost plants is supported by the description of many effector homologues between $B g h$ and $B g t$ [59]. 


\section{Non-adapted pathogens evoke highly specific} transcriptional responses in wheat and barley

The DEGs significantly differing in their regulation between host and nonhost interactions were identified as a sub-set of the general pathogen-regulated DEGs in a two-step statistical approach. These nonhost specific changes in the transcriptome may be the result of: (i) the ability of the adapted isolate to alter the plant gene transcriptome to create an invasion friendly environment, something the non-adapted isolate is unable to do; (ii) suppression of a part of the general plant defense transcriptome by the adapted isolate to allow successful invasion; and/or (iii) specific defense responses evoked by the non-adapted, but not the adapted isolate. One of the key findings of this work is that, in contrast to the general pathogen-regulated genes, the genes differentially expressed between host and nonhost interactions showed only small overlaps between the three cereal pathosystems. This is in line with findings in barley where transcriptional changes associated with different nonhost interactions were generally specific to the corresponding pair of adapted and non-adapted isolates [19]. The observed pathosystem specificity of nonhost-related responses may in part be explained by the different lifestyles of the pathosystems investigated, each having evolved unique strategies for successful invasion and host immunity suppression. Taken together, our data and those from previous studies $[8,19]$ propose PAMPmediated induction of a general defense response, which would be triggered by adapted and non-adapted isolates in all three pathosystems. In a pathosystem specific manner, this general response would then be overlaid by selective suppression, with the non-adapted isolate being unable to sufficiently manipulate the general defense systems of the plant for successful invasion (Fig. 8).

\section{General pathogen-regulated transcription profiles are conserved in wheat and barley}

Having compared the sets of pathogen-responsive genes in wheat and barley to adapted and non-adapted isolates from three distinct pathosystems, we then asked whether the transcriptional profiles had been conserved across these two Triticeae species. For this comparison we identified putative orthologues between wheat and barley taking two approaches; (i) an adjusted reciprocal best blast hit (RBH) method and (ii) creation of orthologue clusters using InParanoid [34]. We found that the transcriptional profiles of the orthologous DEGs between wheat and barley for the general pathogen-regulated genes were very similar. Schreiber et al. [60] undertook a comparative transcriptomics study in the Triticeae, looking at different tissues and developmental conditions, and also found conservation of expression profiles between wheat and barley. There is also evidence that part of the plant response to fungal pathogens is conserved between monocot and dicot species. Humphry et al. [61] described a regulon of antifungal defense in barley and Arabidopsis thaliana, which is co-regulated with known components of the plant defense system (MLO, SNAP34, HvROR2 as homologue of AtPEN1, and HvPDR8 as homologue of AtPEN3). In barley, this highly conserved regulon included 356 ESTs [61], of which, interestingly, 73\% were also found in the list of general pathogen-regulated DEGs of barley presented here (Fig. 2a, Additional file 4: Table S1). In conclusion there exists a core pathogenesisrelated regulon in wheat and barley for the pathosystems investigated.

\section{Nonhost-related transcriptional profiles differ between wheat and barley}

Besides the fact that only relatively small sets of orthologue-matched nonhost-related DEGs were found, comparison of their transcriptional profiles showed no clear conservation between wheat and barley. The apparent lack of nonhost-related co-regulation of gene orthologues may be the result of (i) wheat and barley using an overlapping set of genes that are differently fine-tuned during defense against different non-adapted isolates, (ii) overlapping gene sets of the general defense response being manipulated differently by the adapted isolates in wheat and in barley, each in a manner that is optimal to the requirements of the respective adapted pathogen (Fig. 8), or (iii) the small sets of orthologue-matched nonhost-specific genes representing false positives in one or both species. Option (ii) may seem most plausible, as during co-evolution with their host plant each adapted isolate would have evolved specific effectors to suppress defense and enable invasion of that specific plant species. Although the effector repertoires of closely related pathogen species such as Bgt and Bgh, Mo Br116.5 and Mo TH6772, or $P t$ and $P h$, may overlap to a certain extent, pathogen species-specific effectors might be responsible for evoking different transcriptional responses in their host plant. In our study, the wheat transcriptome responded earlier to the adapted Blumeria isolate than the barley transcriptome, whereas the response to adapted isolates of Magnaporthe and Puccinia was weaker in wheat than in barley. These differences in the transcriptional reprogramming were to some extent mirrored in the cytology. In the host interactions Bgt evoked an earlier plant cellular response in wheat than Bgh in barley, while Mo Br116.5 and Pt on wheat developed more slowly than Mo TH6772 and Ph on barley. The differences in fungal development could explain the distinct gene expression profiles of wheat and barley, further emphasizing the impact of pathogen isolate-specific properties. Option (iii), in the case of the Puccinia pathosystem, appears unlikely because the orthologous gene set 


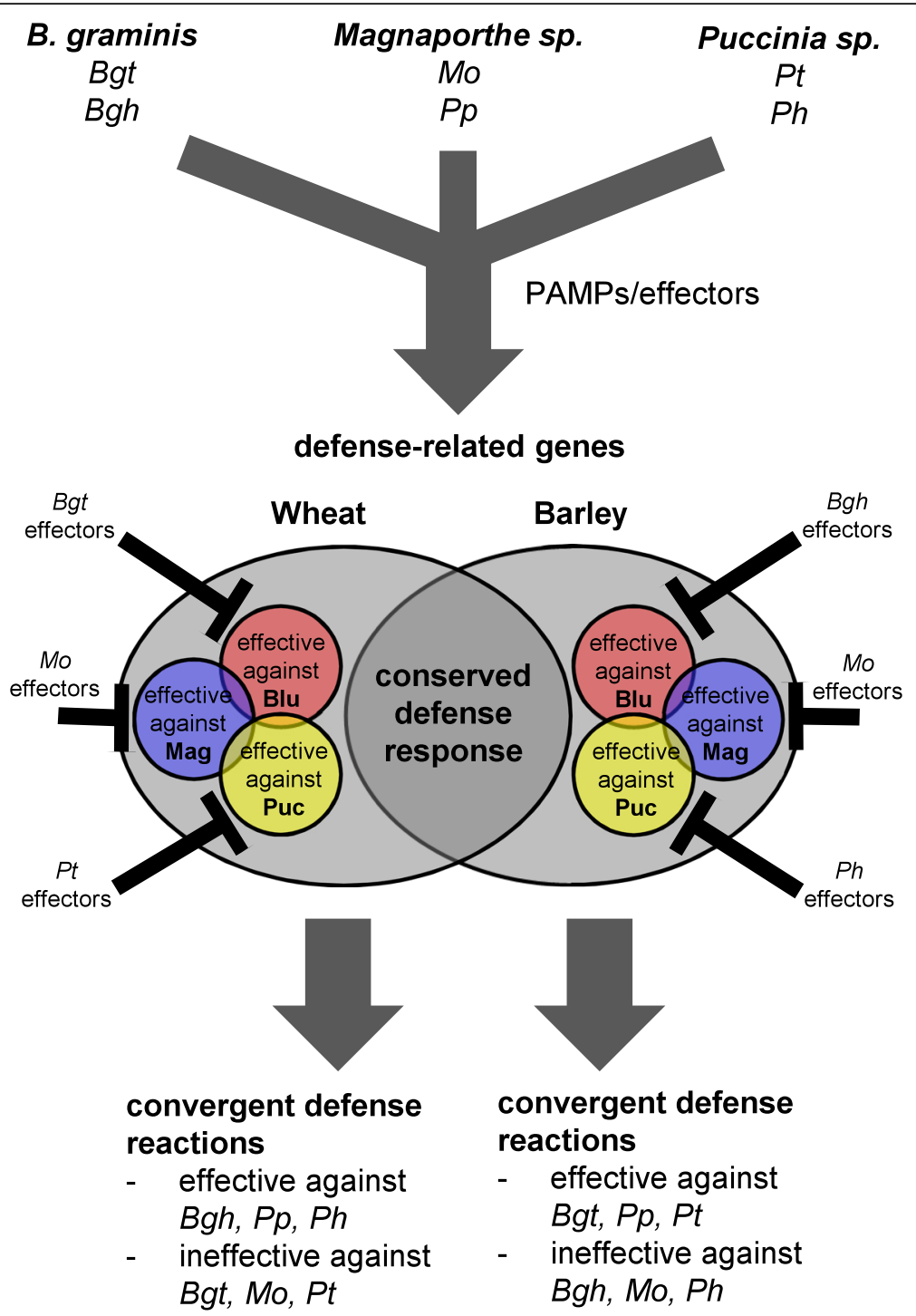

Fig. 8 Model of selective suppression of host defense by different pathogens in two Triticeae species. Adapted and non-adapted isolates of different pathogen genera evoke a similar transcriptional re-programming in wheat and barley. Different parts of this general response are selectively suppressed by the adapted isolates. This results in a specific differential regulation between host and respective nonhost interactions. Although the outcome of defense reactions against the different pathogens is convergent, also in wheat and barley, they are differently effective against the individual isolates (adapted from [19])

is larger and exhibits an obvious trend for enhanced regulation in a pathogen species- but not nonhost statusdependent manner, i.e. $\mathrm{Ph}$ induced stronger transcriptional responses than $P t$, both in wheat and barley (Fig. 7). However, gene orthology does not necessarily predict identical gene function, because neo-functionalization of orthologous genes may occur independently in different plant species [62]. Besides the orthologue mapping, we therefore looked for across species-conserved regulation of genes potentially involved in NHR, by first selecting strictly co-regulated, nonhost-related genes in both wheat and barley, followed by Blast-based analysis of their potential function or gene-family membership (Additional file 11:
Figure S6). This revealed $k$-means subclusters in all three pathosystems, which are clearly co-regulated in a nonhost-specific manner across wheat and barley. Some of these, such as subcluster 4 and 9 in the Blumeria pathosystem (Additional file 11: Figure S6), showed strong or even qualitative differences between hosts and nonhosts. However, there was no evidence for any functional relatedness of shared family membership of these strictly co-regulated genes. This further substantiated the observation, that NHR in wheat and barley probably relies on different essential responses.

Superimposed upon a common, basal pathogen defense response, wheat and barley may have evolved different 
responses to a particular pathogen species after the wheatbarley species split which occurred some 10-14 million years ago [60]. These responses might be triggered by stacks of NLR-type resistance genes [20]. While the adapted isolates are constantly overcoming NLR recognition and are efficiently suppressing basal defense responses, other, gradually less adapted isolates would have lost these capabilities and would remain non-adapted isolates. The expected outcome of such a scenario of gradually independent host-pathogen co-evolution would be a considerable non-overlap of nonhost-differentially regulated genes, as described here. Differences in NHR defense responses have also been reported to be host genotype specific in rice and barley. In rice the regulation of defense genes in response to adapted and non-adapted isolates of Magnaporthe differed between rice cultivars [63]. A similar genotype-specific response was observed for barley, where NHR to $P t$ in different cultivars depended on a different set of genes [13], indicating that different NHR defense responses have even evolved within plant species.

\section{Conclusions}

With respect to the value of NHR for plant resistance breeding, it may be reasonable to consider the transfer of NHR from one species to the other. In barley, the receptor-like kinase $L E M K 1$ was shown to confer NHR to $B g t$, while transient over-expression of $H v L E M K 1$ was found to reduce the establishment of Bgt haustoria in epidermal cells of the wheat cv. Kanzler [64]. Johnston et al. [65] also reported transferring $P h$ resistance from the nonhost species Hordeum bulbosum to H. vulgare. In wheat, the multi-pathogen resistance gene Lr34/Yr18/ Sr57/Pm38 has been shown to enhance resistance against rusts and powdery mildew when expressed in barley, and against $M$. oryzae in rice [66, 67]. Therefore the global transcriptomic data sets generated within this study provide a valuable resource for further investigation aimed at identifying orthologous NHR as well as non-orthologous host susceptibility components in wheat and barley, that upon transfer across cereal species and mutagenesis, respectively, may confer durable host resistance.

\section{Methods}

\section{Cultivation of plants and pathogens and inoculation procedures}

Pathogen inoculation experiments were performed on barley cultivar Vada and on wheat cultivar Renan.

For Blumeria inoculation plants were grown in plastic pots (14 cm diameter) with standard compost soil mixture (from IPK, Gatersleben greenhouse nursery) without fertilization. Seedlings were allowed to grow in a plant climate chamber (NEMA Industrietechnik $\mathrm{GmbH}$ ) at $19{ }^{\circ} \mathrm{C}$ with $65 \%$ relative humidity $(\mathrm{RH})$ during night and $23{ }^{\circ} \mathrm{C}$ with $50 \%$ RH during day with 16 h of photoperiod. Bgh strain $\mathrm{CH} 4.8$ and $B g t$ Swiss field isolate FAL92315 were used to inoculate the plants with a spore density of $50-80$ conidia $\mathrm{mm}^{-2}$ by shaking the spores over the test plants in a settling tower of approximately $60 \times 60 \times 60 \mathrm{~cm}$. Inoculated and non-inoculated control plants were incubated in the same plant climatized room mentioned above with indirect sunlight until epidermal peeling.

The Magnaporthe isolates CD180 from Pennisetum sp., TH6772 from rice and Br116.5 from wheat were kindly provided by Didier Tharreau (CIRAD Montpellier, France), by the institute of Biochemistry, Tamagawa University (Machida-shi, Tokyo, Japan) and by Yukio Tosa (Kobe University, Japan [68]), respectively. Cultivation of plants and fungi as well as inoculum preparation were performed as described before [69]. The conidia suspension was adjusted to 400,000 spores $\mathrm{ml}^{-1}$, diluted $1: 2$ with surfactant $\left(2 \mathrm{~g} \mathrm{l}^{-1}\right.$ gelatin, $1 \mathrm{ml} \mathrm{l}^{-1}$ Tween) to a final concentration of 200,000 conidia $\mathrm{ml}^{-1}$ and sprayinoculated onto plants. For mock-inoculation plants were sprayed with $50 \%$ surfactant solution. During the first $24 \mathrm{~h}$ after inoculation, plants were incubated at $24^{\circ}$ $\mathrm{C}$ and $100 \%$ relative humidity in the dark. Afterwards, they were cultivated under a plastic hood with growing conditions. Preliminary inoculation tests were performed to find adequate isolates for host and nonhost interactions with barley and wheat. Isolate CD180 was confirmed to be non-adapted on different wheat and barley cultivars (unpublished data, [30]). Isolate Br116.5 evoked distinct blast lesions on wheat cv. Renan (Fig. 1a), but extraordinary severe blast symptoms and leaf death on barley. Instead TH6772, nonpathogenic on wheat cv. Renan, evoked a similar disease phenotype of distinct blast lesions on barley cv. Vada (Fig. 1a).

For rust inoculation, fifty seedlings of the barley cultivar Vada and the wheat cultivar Renan were grown in boxes $(37 \times 39 \mathrm{~cm})$. Ten days after sowing, the first leaves were fixed in horizontal position, the adaxial side facing up. Puccinia hordei, 1.2.1 isolate, and Puccinia triticina, BRW96258 isolate, were used for inoculation. For mock inoculation only Lycopodium powder was used. Inoculations were performed with $10 \mathrm{mg}$ of rust urediniospores per box in a settling tower [70]. The inoculated seedlings were incubated overnight in a dew chamber for $10 \mathrm{~h}$ (17$18{ }^{\circ} \mathrm{C}$ ) at $100 \%$ relative humidity and darkness and then transferred to a greenhouse compartment.

\section{Cytological investigations}

To study the microscopic response to Bgh and Bgt, oneweek-old primary leaves were inoculated with 8-10 conidia $\mathrm{mm}^{-2}$ at whole plant level. After inoculation, pots were incubated in the plant climatized room mentioned above until the respective harvesting time point. For visualization of the fungus, leaves were stained with Coomassie solution 
(0.3\% Coomassie R250 stain, 7.5\% Trichloroacetic acid (TCA), 50\% methanol) for 10-15 min, washed and stored in water.

Barley and wheat leaves inoculated with Magnaporthe isolates were prepared for microscopic investigation of early fungal developmental stages as described previously $[69,71]$. Harvested leaves were placed on Whatman paper soaked with $25 \%$ acetic acid in ethanol $(v / v)$. When cleared of chlorophyll, leaves were microscopically examined in water with a DMBRE microscope (Leica Microsystems, Wetzlar, Germany).

For histological analysis of host and nonhost interactions of barley and wheat with Puccinia, two leaf segments, $2-3 \mathrm{~cm}$ long, were excised from the inoculated seedlings at three time points, 12,24 and $36 \mathrm{hpi}$. The leaf segments were fixed and cleared by boiling for $1.5 \mathrm{~min}$ in lactophenol/ethanol $(1: 2 \mathrm{v} / \mathrm{v})$ in a boiling water bath, and left overnight in this mixture at room temperature. Leaf segments were stained in $0.1 \%$ Uvitex 2B (Ciba-Geigy, Switzerland) as described by Hoogkamp et al. [72]. The preparations were examined with an epifluorescence microscope Axiophot (Zeiss, Germany).

\section{Microarray hybridization and data analysis}

For each pathosystem (Blumeria, Magnaporthe or Puccinia) wheat and barley leaf material was harvested at four different time points after inoculation with an adapted or non-adapted isolate or the respective mocktreatment in three independent experiments (giving 3 times 12 samples per plant-pathosystem combination). For Blumeria and Magnaporthe interactions, the abaxial epidermis of 20-200 inoculated primary leaves was sampled, while in case of the Puccinia interactions whole leaf material of 40 plants was harvested. RNA from all samples was extracted using the RNeasy Plant Mini Kit (Qiagen, Hilden, Germany). DNA was eliminated from RNA samples by on-column digestion with the RNaseFree DNase Set (Qiagen). In the case of Puccinia treated samples, the Ambion ${ }^{\circ}$ TURBO DNA-free ${ }^{\mathrm{Tm}}$ DNase Kit was used for DNA elimination. After passing quality control (using Agilent 2100 Bioanalyzer, Agilent Technologies, Inc.) RNA was hybridized to Agilent $44 \mathrm{k}$ oligonucleotide arrays using One Color MicroarrayBased Gene Expression Analysis- Low Input Quick Amp Labeling, v 6.5, as recommended (Agilent Technologies). The Agilent wheat Expression Microarray (design ID 22297, Agilent technologies, Santa Clara, USA) and a custom barley microarray SCRI_Hv35_44k_v1 (Agilent design ID 20599) [73-75] were used. Raw microarray data were deposited at ArrayExpress [76, 77] (www.ebi.ac.uk/arrayexpress, accession numbers: E-MTAB-2916 for barley-Blumeria, E-MTAB-3803 for wheat-Blumeria, E-MTAB-5634 for barley-Magnaporthe, E-MTAB-5635 for wheat-Magnaporthe, E-MTAB-5655 for barley-Puccinia, E-MTAB-5656 for wheat-Puccinia).

Microarray data analyses were performed on each plantpathogen combination separately but following the same workflow. Raw microarray data were background-corrected and quantile normalized using GeneSpring GX software (Agilent Technologies). To identify possible outlier samples, principal component analysis and clustering of the data was performed. In the case of the barley-Puccinia interaction, this revealed that samples of one biological replicate did not cluster with the other two replicates (Additional file 14: Figure S8). Therefore, the samples of this single experiment were excluded from further data analyses. For statistical analysis, the mean signal intensities of each time-treatment combination were calculated. Subsequently, data were filtered for probes flagged 'detected' in at least one of the 12 time-treatment combinations. On the filtered data, a combination of three statistical approaches were applied to identify probes with significant regulation in host or nonhost interactions compared to control treatment. The first approach was a paired t-test on the differences between host vs. control and nonhost vs. control taking the averages across the investigated time points ('static' approach). Probes that passed the statistical criteria (fold change $\geq 2$ or $\leq-2, \alpha \leq 0.05$ after Benjamini-Hochberg-correction) were summarized to a non-redundant list. In parallel, in a second and third approach 'single-time point' analyses were performed using One Way ANOVA with post hoc Tukey HSD or an unpaired t-test, respectively. In each approach probes significantly regulated in host vs. control or nonhost vs. control (fold change $\geq 2$ or $\leq-2, \alpha \leq 0.05$ ) at the different time points were pooled. Only the probes that passed both single-time point approaches were added to the list resulting from the first, static approach to give a non-redundant list of 'general pathogen-regulated probes'. To identify probes with a significantly different regulation in host compared to nonhost interaction, a One Way ANOVA with post hoc Tukey HSD was applied to the list of general pathogen-regulated probes. Probes showing significant differential expression in host and nonhost interaction (fold change $\geq 2$ or $\leq-2, \alpha \leq 0.05$ ) for at least one time point investigated were summarized to a non-redundant list of 'nonhost-related differentially regulated' probes. For each plant-pathogen interaction the lists of probes found to be 'general pathogen-regulated' and 'differentially regulated between host and nonhost interaction' are given in Additional file 4: Table S1 and Additional file 6: Table S2, respectively (according to Venn diagrams in Fig. 2). The reliability of the array-derived transcriptional data were verified by RT-qPCR with primer pairs for a number of (randomly chosen) Magnaporthe-regulated transcripts and revealed good agreement between results from both methods (Additional file 15: Figure S9). Primer used in this analysis listed in Additional file 16: Table S6. 


\section{Meta analyses of transcriptome data}

Probes of the wheat and the barley microarrays were assigned to unigenes of the DFCI Triticum aestivum Gene Index (TaGI) database $[78,79]$ or the HarvEST database (HarvEST:Barley, v. 1.83) [80], respectively. Sequences were re-annotated using Basic Local Alignment Tool (BLAST) $[81,82]$ with the databases of the National Center for Biotechnology Information (NCBI) [83] and the Blast2Go software [84]. The oligonucleotide sequences of the barley microarray were also assigned to the contigs of the barley genome published by the International Barley Genome Sequencing Consortium (IBSC) [85]. However, many oligonucleotides were assigned redundantly to single cds sequences of IBSC, so that a transcriptome analysis based on these cds sequences would have required a previous data reduction. To circumvent the loss of possibly meaningful data, the barley transcriptome data were analyzed based on the unigene sequences to which the microarray was originally designed [73-75]. Unigene sequences present on the barley and the wheat microarray were assigned to functional 'MapMan' categories using Mercator pipeline [86, 87]. Over-representation analyses (ORA) of functional categories in lists of regulated unigenes were performed with the web-based MapMan ORA tool [35, 36]. For orthologue identification between wheat and barley unigenes, a reciprocal best hit (RBH) analysis between the TaGI and the HarvEST unigenes was performed. Additionally, orthologue clusters of the TaGI wheat unigenes and the barley cds sequences of IBSC (2012) were determined using InParanoid [34]. The results of both approaches were combined to a matching table linking $57.8 \%$ of the wheat to $38.0 \%$ of the barley probes (Additional file 7: Table S3). In order to test whether the overlap between wheat and barley general pathogenregulated DEGs was larger than by chance, (i) the same number of wheat (barley) probes that were significant were sampled translated into their respective orthologues and compared with the barley (wheat) list of significant genes. Alternatively (ii), the wheat (barley) probes declared significant that had an orthology in barley (wheat) were counted and the same number of wheat (barley) genes featuring an orthologue was sampled translated into their orthologues and compared to the barley (wheat) list of genes (Additional file 8: Data S1). In each case, these analyses revealed the numbers of matched orthologues within random gene sets to be lower than within the identified DEGs. Hierarchical and $k$-means clusterings were performed using the software Genesis (version 1.8) [88].

\section{Additional files}

Additional file 1: Figure S1. Quantitative cytology of wheat and barley interactions with Blumeria isolates. Wheat cv. Renan and barley cv. Vada were inoculated with Blumeria graminis f. sp. tritici (Bgt) and Blumeria graminis f. sp. hordei (Bgh). At timepoints indicated interaction sites with fungal appressoria were cytologically evaluated for presence of elongating secondary hyphae $(\mathrm{ESH})$. In addition, the autofluorescence response of the plant was evaluated and assigned to categories indicated. Columns represent mean category percentages of 100 interactions sites counted from four leaves in two biological experiments. (PDF $58 \mathrm{~kb}$ )

Additional file 2: Figure S2. Quantitative cytology of wheat and barley interactions with Magnaporthe isolates. Wheat cv. Renan and barley cv. Vada were inoculated with Magnaporthe oryzae (isolate Br116.5 or TH6772, respectively) and Pyricularia penniseticola (isolate CD180). At timepoints indicated interaction sites with fungal conidiospores were cytologically evaluated for presence of germ tube, appressorium and fungal growth inside the attacked epidermal cell. In addition, at 24 and $48 \mathrm{~h}$ after inoculation the autofluorescence response of the plant was evaluated and assigned to categories indicated. Columns represent mean category percentages of at least 100 interactions sites counted from two to four leaves in three (wheat) and two (barley) biological experiments. (PDF $88 \mathrm{~kb}$ )

Additional file 3: Figure S3. Quantitative cytology of wheat and barley interactions with Puccinia isolates. Wheat cv. Renan and barley cv. Vada were inoculated with Puccinia triticina and Puccinia hordei. At 12, 24 and $36 \mathrm{~h}$ past inoculation (hpi) interaction sites with fungal appressoria were cytologically evaluated for the developmental stage of the fungus (appressorium, substomatal vesicle, infection hyphae and haustorial mother cell). At 48 hpi infection units that had formed a haustorial mother cell were assigned for categories of further development as indicated. Columns represent category percentages of approx. 100 interactions sites of two investigated leaves. (PDF 74 kb)

Additional file 4: Table S1. Lists of wheat and barley genes general regulated after pathogen inoculation (lists of the subsets according to Venn diagrams in Fig. 2a). Signal values are an output of the GeneSpring GX software and given as log base 2 values. Fold changes are calculated accordingly. (XLSX 22727 kb)

Additional file 5: Figure S4. Numbers of DEGs found to be up- or downregulated in host or nonhost interactions compared to mock inoculations (according to average log fold changes of host vs. control and nonhost vs. control at different time points). (PDF $24 \mathrm{~kb}$ )

Additional file 6: Table S2. Lists of wheat and barley genes differentially regulated in host and nonhost interactions with Blumeria, Magnaporthe and Puccinia (lists of the subsets according to Venn diagrams in Fig. 2b). Signal values are an output of the GeneSpring GX software and given as log base 2 values. Fold changes are calculated accordingly. (XLSX $3027 \mathrm{~kb})$

Additional file 7: Table S3. Linking of wheat and barley orthologue candidates. (XLSX $2951 \mathrm{~kb}$ )

Additional file 8: Data S1. Simulations of orthologue assignment within random wheat (barley) gene sets to general pathogen regulated barley (wheat) DEGs showing that the overlap found between wheat and barley general pathogen-regulated DEGs was larger than by chance. (PDF $10 \mathrm{~kb}$ )

Additional file 9: Table S4. IDs, median-centered intensity values and Blast annotation of orthologue-matched DEGs represented in the heat maps of Figs. 4, 5, 6 and 7. (XLSX 1559 kb)

Additional file 10: Figure S5. Numbers of genes found to be differentially regulated between host and nonhost interactions at different time points in wheat or barley after inoculation with adapted and non-adapted isolates of Blumeria, Magnaporthe and Puccinia (according to statistical analysis of microarray data). (PDF 51 kb)

Additional file 11: Figure S6. Results of $k$-means clustering followed by hierarchical clustering on the nonhost-related DEGs of wheat and barley in each pathosystem. In an independent approach to identify coregulated orthologues, a $k$-means clustering followed by a hierarchical clustering of individual $k$-means cluster members was carried out for both cereal species and respective interactions. For this, the normalized signal intensity values of host and nonhost DEGs (Fig. 2b) of the respective pathosystem were median-centered separately for wheat and barley in Genesis software (release 1.8.0, [88]). The median-centered signal values of both species were pooled for each pathosystem to form a single list, 
and a k-means clustering analysis was done in Genesis with default settings. Individual $k$-means clusters were then hierarchical clustered in Genesis with Pearson correlation, average linkage settings. To identify co-regulated regulons, sub-clusters with the occurrence of both wheat and barley probes (left clustering image; nodes with sub-clusters highlighted in color) were manually identified and extracted. A zoomed in image of these sub-clusters is shown on the right. Please note the occurrence of both wheat (A_99_) and barley (CUST_) probe IDs in the same node. In order to determine the gene function relation among these co-regulated wheat and barley DEGs, MapMan (3.5.1R2) and BlastN analyses (default parameters, BLASTN 2.2.29+) were carried out. (PDF $3400 \mathrm{~kb}$ )

Additional file 12: Table S5. IDs, median-centered intensity values and MapMan annotation of $k$-means and hierarchical clustering subclusters (Additional file 11: Figure S6), and results of BlastN analysis within the subclusters. (XLSX $12681 \mathrm{~kb})$

Additional file 13: Figure S7. Overrepresentation analysis of functional MapMan categories within the intersections of general pathogen regulated wheat and barley DEGs (a) and DEGs with differential expression between host and nonhost interaction (b) found for the three pathosystems Blumeria, Magnaporthe and Puccinia. Over- or underrepresentation and statistical significance according to Fisher Exact Test were calculated with MapMan ORA tool $[35,36]$. All probes assigned to MapMan BINs were taken as reference. The log ratio of presentation in the gene set and in the reference is shown for BINs found to be significant (BINs comprising 5 or less probes were excluded). (PDF $14 \mathrm{~kb}$ )

Additional file 14: Figure S8. Clusterings of Puccinia microarray data leading to the decision to exclude one of three biological barley replicates. Ideally, the replications $(1,2,3)$ from the same treatment (mock, host, nonhost) and time point $(12,24,36$ and $48 \mathrm{hpi})$ should cluster together. The clustering of chips was very clear in case of wheat/Puccinia interaction (a). In case of barley/Puccinia this was not the case: 7 out of 12 chips from replication 1 did not cluster into their respective subclades (b) (coloured arrows indicate chips out of their respective clusters). Therefore the complete replication 1 of barley/ Puccinia interactions was omitted from the final analysis, resulting in a great enrichment of host/nonhost differentially regulated genes (11 according to analysis with all 3 replicates, 1824 according to analysis without replicate 1). (PDF $1063 \mathrm{~kb}$ )

Additional file 15: Figure S9. Validation of microarray gene expression profiles by quantitative reverse transcription PCR (qRT-PCR). For selected candidate genes in the barley-Magnaporthe pathosystem, normalized signal intensities resulting from the microarray analyses (data points show averages and standard errors of three biological replicates) were compared with relative transcript abundancies resulting from qRT-PCR analysis of an independent experiment (bars show averages and standard deviations of two technical replicates). qRT-PCR was performed as described [69]. Asterisks indicate time points for which microarray analyses showed a significant differential expression during nonhost interaction compared to host interaction. In $x$-marked samples the respective candidate gene transcript was not detectable by qRT-PCR. (PDF $152 \mathrm{~kb})$

Additional file 16: Table S6. Primer combinations used for validation of microarray gene expression profiles by qRT- PCR (Additional file 15: Figure S9). (PDF $21 \mathrm{~kb}$ )

\section{Abbreviations}

Bgh: Blumeria graminis f. sp. hordei; Bgt: Blumeria graminis f. sp. tritici; BLAST: Basic local alignment tool; DEG: Differentially expressed gene; ETI: Effector-triggered immunity; HMC: Haustorial mother cell; hpi: Hours past inoculation; HR: Hypersensitive response; Mo: Magnaporthe oryzae; NHR: Nonhost resistance; ORA: Over-representation analysis; PAMP: Pathogen-associated molecular pattern; Ph: Puccinia hordei; Pp: Pyricularia penniseticola; PRR: Pathogen recognition receptor; Pt: Puccinia triticina; PTI: PAMP-triggered immunity; RBH: Reciprocal best hit; RH: Relative humidity; RLK: Receptor-like kinase

\section{Acknowledgements}

The authors would like to thank Didier Tharreau (CIRAD Montpellier) and Yukio Tosa (Kobe University) for providing Magnaporthe isolates, Renate Schubert (RWTH Aachen University) and Ruth MacCormack (John Innes Centre) for technical assistance, Jenny Morris (James Hutton Institute) for the physical array processing, Stefanie Lück (IPK Gatersleben) for help with blast analysis of $k$-means clusters, and Pamela Abbruscato (Parco Tecnologico Padano) for helpful discussions.

\section{Funding}

This work was part of the project TritNONHOST funded by the ERA-NET Plant Genomics supported by The German Research Foundation (DFG, grant nos. SCHW848/3-1 and SCHA631/5-1), by Netherlands Genomics Initiative / Netherlands Organisation for Scientific Research (NGI/NOW, grant no. 855.50.015), and by the UK Biotechnology and Biological Sciences Research Council (BBSRC, award BB/G024987/1). The funding bodies had no role in the design of the study and collection, analysis, and interpretation of data and in writing the manuscript.

\section{Availability of data and materials}

The raw microarray datasets generated and analyzed in this study are available in the ArrayExpress database (www.ebi.ac.uk/arrayexpress) [76, 77] under accession numbers E-MTAB-2916, E-MTAB-3803, E-MTAB-5634, E-MTAB-5635, E-MTAB-5655 and E-MTAB-5656. The normalized gene expression values of differentially expressed probes are given in Additional file 4: Table S1 and Additional file 6: Table S2.

\section{Authors' contributions}

$\mathrm{RD}$ and JR performed most of the experiments and data analyses; RA, FLS, GM and PEH performed experiments; FLS, SR and BU analyzed data; MB and BU contributed new MapMan annotation; US, PS, REN and LB conceived the project and original research plans and supervised the experiments; RD drafted the manuscript with contributions of all the authors; LB, PS and US supervised and complemented the writing. All authors read an approved the final manuscript.

\section{Ethics approval and consent to participate}

Not applicable.

\section{Consent for publication}

Not applicable.

\section{Competing interests}

The authors declare that they have no competing interests.

\section{Publisher's Note}

Springer Nature remains neutral with regard to jurisdictional claims in published maps and institutional affiliations.

\section{Author details \\ 'Department of Plant Physiology, RWTH Aachen University, 52056 Aachen, Germany. ${ }^{2}$ Leibniz-Institute of Plant Genetics and Crop Plant Research, 06466 Gatersleben, Germany. ${ }^{3}$ Department of Disease and Stress Biology, John Innes Centre, Norwich Research Park, Colney Lane, Colney, Norwich, Norfolk NR4 7UH, UK. ${ }^{4}$ Plant Breeding, Graduate School for Experimental Plant Sciences, Wageningen University \& Research, Droevendaalsesteeg 1, 6708, $\mathrm{PB}$, Wageningen, the Netherlands. Institute of Botany and Molecular Genetics, BioSC, RWTH Aachen University, 52056 Aachen, Germany. ${ }^{6}$ The James Hutton Institute, Invergowrie, Dundee, Scotland DD2 5DA, UK. ${ }^{7} \mathrm{NIAB}$, Huntingdon Road, Cambridge CB3 OLE, UK. ${ }^{8}$ Present address: Molecular microbiology, John Innes Centre, Norwich Research Park, Norwich NR4 7UH, UK. ${ }^{9}$ Present address: Biodiversity and Integrated Gene Management Program (BIGM), International Center for Agriculture Research in the Dry Areas, Rabat, Morocco. ${ }^{10}$ Present address: Seed and Plant Improvement Research Department, Khorasan Razavi Agricultural and Natural Resources Research and Education Center, AREEO, Mashhad, Iran.}

Received: 14 June 2017 Accepted: 15 November 2017 Published online: 04 December 2017

\section{References}

1. Feuillet C, Muehlbauer GJ, editors. Genetics and genomics of the Triticeae. Dordrecht Heidelberg London New York: Springer; 2009.

2. Food and Agriculture Organization of the United Nations. FAOSTAT 2014 Available at http://www.fao.org/faostat/en/\#data/QC. 
3. Oerke E-C. Crop losses to pests. J Agric Sci. 2006;144:31-43. Available from: http://journals.cambridge.org/article_S0021859605005708

4. Li Y, Huang F, Lu Y, Shi Y, Zhang M, Fan J, et al. Mechanism of plant-microbe interaction and its utilization in disease-resistance breeding for modern agriculture. Physiol Mol Plant Pathol. 2013;83:51-8. Available from: http:/www.sciencedirect. com/science/article/pii/S0885576513000301

5. Lee S, Hutton S, Whitaker V. Mini review: potential applications of nonhost resistance for crop improvement. Front Plant Sci. 2016;7:997. Available from: http://www.frontiersin.org/plant_biotic_interactions/10.3389/fpls.2016.00997/ abstract

6. Mysore KS, Ryu C-M. Nonhost resistance: how much do we know? Trends Plant Sci. 2004;9:97-104. Available from: http://dx.doi.org/10.1016/j.tplants. 2003.12.005

7. Jones JDG, Dangl JL. The plant immune system. Nature. 2006;444:323-9. Available from: http://dx.doi.org/10.1038/nature05286

8. Niks RE, Marcel TC. Nonhost and basal resistance: how to explain specificity? New Phytol. 2009;182:817-28. Available from: http://dx.doi.org/10.1111/j. 1469-8137.2009.02849.x

9. Dangl JL, Horvath DM, Staskawicz BJ. Pivoting the plant immune system from dissection to deployment. Science. 2013;341:746-51. Available from: http://science.sciencemag.org/content/341/6147/746

10. Heath MC. Nonhost resistance and nonspecific plant defenses. Curr Opin Plant Biol. 2000;3:315-9. Available from: http://www.sciencedirect.com/ science/article/pii/S136952660000087X

11. Strugala $R$, Delventhal $R$, Schaffrath U. An organ-specific view on nonhost resistance. Front Plant Sci. 2015;6:526. Available from: http://www.frontiersin. org/plant_biotic_interactions/10.3389/fpls.2015.00526/abstract

12. Lee H-A, Lee H-Y, Seo E, Lee J, Kim S-B, Oh S, et al. Current understandings on plant nonhost resistance. Mol Plant-Microbe Interact. 2017;30:5-15. Available from: http://apsjournals.apsnet.org/doi/10.1094/MPMI-10-16-0213$C R$

13. Jafary H, Albertazzi G, Marcel TC, Niks RE. High diversity of genes for nonhost resistance of barley to heterologous rust fungi. Genetics. 2008;178: 2327-39. Available from: http://dx.doi.org/10.1534/genetics.107.077552

14. Aghnoum R, Niks RE. Specificity and levels of nonhost resistance to nonadapted Blumeria graminis forms in barley. New Phytol. 2010;185:27584. Available from: http://dx.doi.org/10.1111/j.1469-8137.2009.03039.x

15. Niks RE, Qi X, Marcel TC. Quantitative resistance to biotrophic filamentous plant pathogens: concepts, misconceptions, and mechanisms. Annu Rev Phytopathol. 2015;53:445-70. Available from: http://dx.doi.org/10.1146/ annurev-phyto-080614-115928

16. Heath MC. Non-host resistance to plant pathogens: nonspecific defense or the result of specific recognition events? Physiol Mol Plant Pathol. 2001;58:53-4 Available from: http://inkinghub.elsevier.com/retrieve/pii/S0885576501903194

17. Sindhu A, Chintamanani S, Brandt AS, Zanis M, Scofield SR, Johal GS. A guardian of grasses: specific origin and conservation of a unique diseaseresistance gene in the grass lineage. Proc Natl Acad Sci U S A. 2008;105: 1762-7. Available from: http://www.pnas.org/content/105/5/1762.full.

18. Schweizer P. Nonhost resistance of plants to powdery mildew-new opportunities to unravel the mystery. Physiol Mol Plant Pathol. 2007; 70:3-7. Available from: http://www.sciencedirect.com/science/article/ pii/S088557650700063X

19. Zellerhoff N, Himmelbach A, Dong W, Bieri S, Schaffrath U, Schweizer P. Nonhost resistance of barley to different fungal pathogens is associated with largely distinct, quantitative transcriptional responses. Plant Physiol. 2010;152:2053-66.

20. Schulze-Lefert P, Panstruga R. A molecular evolutionary concept connecting nonhost resistance, pathogen host range, and pathogen speciation. Trends Plant Sci. 2011;16:117-25. Available from: http://dx.doi.org/10.1016/j.tplants. 2011.01 .001

21. Newton A, Flavell A, George T, Leat P, Mullholland B, Ramsay L, et al. Crops that feed the world 4. Barley: a resilient crop? Strengths and weaknesses in the context of food security. Food Secur. 2011:3:141-78. Available from: http://dx.doi.org/10.1007/s12571-011-0126-3

22. Shiferaw B, Smale M, Braun H-J, Duveiller E, Reynolds M, Muricho G. Crops that feed the world 10. Past susuccess and future challenge to the role played by wheat in global food security. Food Secur. 2013;3:141-78. Available from: http://dx.doi.org/10.1007/s12571-011-0126-3

23. Malaker PK, Barma NCD, Tewari TP, Collis WJ, Duveiller E, Singh PK, et al. First report of wheat blast caused by Magnaporthe oryzae pathotype triticum in Bangladesh. Plant Dis. 2016;100:2330. Available from: http://dx. doi.org/10.1094/PDIS-05-16-0666-PDN
24. Lyngkjaer MF, Carver TL. Induced accessibility and inaccessibility to Blumeria graminis f.Sp. hordei in barley epidermal cells attacked by a compatible isolate. Physiol Mol Plant Pathol. 1999;55:151-62.

25. Bolton MD, Kolmer JA, Garvin DF. Wheat leaf rust caused by Puccinia triticina. Mol Plant Pathol. 2008;9:563-75. Available from: http://dx.doi.org/10 1111/j.1364-3703.2008.00487.x

26. Feuillet C, Muehlbauer GJ. Genetics and genomics of the Triticeae. Plant Genetics and Genomics: Crops and Models. vol. 7. New York: SpringerVerlag; 2009.

27. Ribot C, Hirsch J, Balzergue S, Tharreau D, Nottéghem J-L, Lebrun M-H, et al. Susceptibility of rice to the blast fungus, Magnaporthe grisea. J Plant Physiol. 2008;165:114-24. Available from: http://dx.doi.org/10.1016/j.jplph.2007.06.013

28. Tosa $Y$, Shishiyama J. Defense reactions of barley cultivars to an inappropriate forma specialis of the powdery mildew fungus of gramineous plants. Can J Bot. 1984;62:2114-7. Available from: http://www. nrcresearchpress.com/doi/abs/10.1139/b84-287

29. Neu C, Keller B, Feuillet C. Cytological and molecular analysis of the Hordeum vulgare-Puccinia triticina nonhost interaction. Mol Plant-Microbe Interact. 2003;16:626-33. Available from: http://dx.doi.org/10.1094/MPMI. 2003.16.7.626

30. Zellerhoff N, Jarosch B, Groenewald JZ, Crous PW, Schaffrath U. Nonhost resistance of barley is successfully manifested against Magnaporthe grisea and a closely related Pennisetum-infecting lineage but is overcome by Magnaporthe oryzae. Mol Plant-Microbe Interact. 2006;19:1014-22. Available from: http://dx.doi.org/10.1094/MPMI-19-1014

31. Klaubauf S, Tharreau D, Fournier E, Groenewald JZ, Crous PW, de Vries RP, et al. Resolving the polyphyletic nature of Pyricularia (Pyriculariaceae). Stud Mycol. 2014;79:85-120. Available from: http://www.sciencedirect.com/ science/article/pii/S0166061614000104

32. Mayama S, Shishiyama J. Localized accumulation of fluorescent and u.V. absorbing compounds at penetration sites in barley leaves infected with Erysiphe graminis hordei. Physiol Plant Pathol. 1978;13:347-54. Available from: http://www.sciencedirect.com/science/article/pii/0048405978900516

33. Koga H, Zeyen RJ, Bushnell WR, Ahlstrand GG. Hypersensitive cell death, autofluorescence, and insoluble silicon accumulation in barley leaf epidermal cells under attack by Erysiphe graminis f. sp. hordei. Physiol Mol Plant Pathol. 1988;32:395-409. Available from: http://www.sciencedirect. com/science/article/pii/S088557658880033X

34. Ostlund G, Schmitt T, Forslund K, Köstler T, Messina DN, Roopra S, et al. InParanoid 7: new algorithms and tools for eukaryotic orthology analysis. Nucleic Acids Res. 2010;38:D196-203. Available from: http://dx.doi.org/10. 1093/nar/gkp931

35. Over Representation Analysis Tool for supported MapMan organisms. Available from: http://mapman.gabipd.org/web/guest/pageman

36. Usadel B, Nagel A, Steinhauser D, Gibon Y, Bläsing OE, Redestig H, et al. PageMan: an interactive ontology tool to generate, display, and annotate overview graphs for profiling experiments. BMC Bioinf. 2006;7:535. Available from: http://dx.doi.org/10.1186/1471-2105-7-535

37. Douchkov D, Lueck S, Hensel G, Kumlehn J, Rajaraman J, Johrde A, et al. The barley (Hordeum Vulgare) cellulose synthase-like D2 gene (HvCsID2) mediates penetration resistance to host-adapted and nonhost isolates of the powdery mildew fungus. New Phytol. 2016;212:421-33. Available from: http://dx.doi.org/10.1111/nph.14065

38. Chowdhury J, Schober MS, Shirley NJ, Singh RR, Jacobs AK, Douchkov D, et al. Down-regulation of the glucan synthase-like 6 gene (HVGs/6) in barley leads to decreased callose accumulation and increased cell wall penetration by Blumeria graminis f. sp. hordei. New Phytol. 2016;n/a-n/a. Available from: http://dx.doi.org/10.1111/nph.14086

39. Chowdhury J, Henderson M, Schweizer P, Burton RA, Fincher GB, Little A. Differential accumulation of callose, arabinoxylan and cellulose in nonpenetrated versus penetrated papillae on leaves of barley infected with Blumeria graminis $\mathrm{f}$. sp. hordei. New Phytol. 2014;204:650-60. Available from: http://dx.doi.org/10.1111/ nph. 12974

40. Caldo RA, Nettleton D, Wise RP. Interaction-dependent gene expression in Mla-specified response to barley powdery mildew. Plant Cell. 2004;16:2514-28. American Society of Plant Biologists. Available from: http://www.ncbi.nlm.nih. gov/pmc/articles/PMC520949/

41. Zhang H, Sreenivasulu N, Weschke W, Stein N, Rudd S, Radchuk V, et al. Large-scale analysis of the barley transcriptome based on expressed sequence tags. Plant J. 2004;40:276-90. Available from: http://dx.doi.org/10. $1111 / j .1365-313 X .2004 .02209 . x$ 
42. Dörmann P, Kim H, Ott T, Schulze-Lefert P, Trujillo M, Wewer V, et al. Cellautonomous defense, re-organization and trafficking of membranes in plant-microbe interactions. New Phytol. 2014;204:815-22. Available from: http://dx.doi.org/10.1111/nph.12978

43. Hao Y, Wang T, Wang K, Wang X, Fu Y, Huang L, et al. Transcriptome analysis provides insights into the mechanisms underlying wheat plant resistance to stripe rust at the adult plant stage. PLoS one. 2016;11: e0150717. Public Library of Science. Available from: https://doi.org/10.1371/ journal.pone.0150717

44. Lipka U, Fuchs R, Lipka V. Arabidopsis non-host resistance to powdery mildews. Curr Opin Plant Biol. 2008;11:404-11. Available from: http://www. sciencedirect.com/science/article/pii/S1369526608000745

45. Dodds PN, Rathjen JP. Plant immunity: towards an integrated view of plantpathogen interactions. Nat Rev Genet. 2010;11:539-48. Available from: http://dx.doi.org/10.1038/nrg2812

46. Thomma BPHJ, Nürnberger T, Joosten MHAJ. Of PAMPs and effectors: the blurred PTI-ETI dichotomy. Plant Cell. 2011;23:4-15. Available from: http://dx. doi.org/10.1105/tpc.110.082602

47. Hanzalová A, Dumalasová V, Sumíková T, Bartoš P. Rust resistance of the French wheat cultivar Renan. Czech J Genet Plant Breed. 2007;43:53-60.

48. Dardick C, Ronald P. Plant and animal pathogen recognition receptors signal through non-RD kinases. PLoS Pathog. 2006;2:e2. Available from: http://dx.plos.org/10.1371\%2Fjournal.ppat.0020002

49. Macho AP, Zipfel C. Plant PRRs and the activation of innate immune signaling. Mol Cell. 2014;54:263-72. Available from: http://www. sciencedirect.com/science/article/pii/S1097276514002615

50. Schnepf V, Vlot AC, Kugler K, Hückelhoven R. Barley susceptibility factor RACB modulates transcript levels of signalling protein genes in compatible interaction with Blumeria graminis f.sp. hordei. Mol. Plant Pathol. 2017;n/a-n/ a. Available from: http://dx.doi.org/10.1111/mpp.12531

51. Dixon RA, Paiva NL. Stress-induced phenylpropanoid metabolism. Plant Cell. 1995;7:1085-97. Available from: http://dx.doi.org/10.1105/tpc.7.7.1085

52. Hammerschmidt R. Induced disease resistance: how do induced plants stop pathogens? Physiol Mol Plant Pathol. 1999;55:77-84. Available from: http:// www.sciencedirect.com/science/article/pii/S0885576599902151

53. Elliston J, Kuc J, Williams EB. A comparative study of the development of compatible, incompatible, and induced incompatible interactions between Colletotrichum spp. and Phaseolus vulgaris. J Phytopathol. 1976;87:289-303. Available from: http://dx.doi.org/10.1111/j.1439-0434.1976.tb01735.x

54. Showalter AM, Bell JN, Cramer CL, Bailey JA, Varner JE, Lamb CJ. Accumulation of hydroxyproline-rich glycoprotein mRNAs in response to fungal elicitor and infection. Proc Natl Acad Sci U S A. 1985;82:6551-5. Available from: http://www.pnas.org/content/82/19/6551.abstract

55. Van Sumere CF, Preter CVS-D, Ledingham GA. Cell-wall-splitting enzymes of Puccinia graminis var. tritici. Can J Microbiol. 1957;3:761-70. NRC Research Press. Available from: http://dx.doi.org/10.1139/m57-086

56. Pryce-Jones E, Carver T, Gurr SJ. The roles of cellulase enzymes and mechanical force in host penetration by Erysiphe graminis f.sp.hordei. Physiol Mol Plant Pathol. 1999;55:175-82. Available from: http://www.sciencedirect. com/science/article/pii/S0885576599902229

57. Jung Y-H, Jeong S-H, Kim SH, Singh R, Lee J, Cho Y-S, et al. Secretome analysis of Magnaporthe oryzae using in vitro systems. Proteomics. 2012;12: 878-900. Available from: http://dx.doi.org/10.1002/pmic.201100142

58. Tsurushima T, Nakayashiki H, Tosa Y, Mayama S. Pathogenicity-related compounds produced by blast fungus. In: Wang G-L, Valent B, editors. Adv. Genet. Genomics Control Rice Blast Dis. Netherlands: Springer; 2009. p. 24755. Available from: http://dx.doi.org/10.1007/978-1-4020-9500-9_25.

59. Wicker T, Oberhaensli S, Parlange F, Buchmann JP, Shatalina M, Roffler S, et al. The wheat powdery mildew genome shows the unique evolution of an obligate biotroph. Nat Genet. 2013;45:1092-6. Available from: http://dx. doi.org/10.1038/ng.2704

60. Schreiber AW, Sutton T, Caldo RA, Kalashyan E, Lovell B, Mayo G, et al. Comparative transcriptomics in the Triticeae. BMC Genomics. 2009;10:285. Available from: http://dx.doi.org/10.1186/1471-2164-10-285

61. Humphry M, Bednarek P, Kemmerling B, Koh S, Stein M, Göbel U, et al. A regulon conserved in monocot and dicot plants defines a functional module in antifungal plant immunity. Proc Natl Acad Sci U S A. 2010;107: 21896-901. Available from: http://dx.doi.org/10.1073/pnas.1003619107

62. Gabaldon T, Koonin EV. Functional and evolutionary implications of gene orthology. Nat Rev Genet. 2013;14:360-6. Available from: http://dx.doi.org/ $10.1038 /$ nrg3456
63. Faivre-Rampant O, Thomas J, Allegre M, Morel J-B, Tharreau D, Notteghem $\mathrm{J}-\mathrm{L}$, et al. Characterization of the model system rice-Magnaporthe for the study of nonhost resistance in cereals. New Phytol. 2008;180:899-910. Available from: http://dx.doi.org/10.1111/j.1469-8137.2008.02621.x

64. Rajaraman J, Douchkov D, Hensel G, Stefanato FL, Gordon A, Ereful N, et al. An $\mathrm{LRR} /$ malectin receptor-like kinase mediates resistance to non-adapted and adapted powdery mildew fungi in barley and wheat. Front Plant Sci. 2016;7:1836 Available from: http://journal.frontiersin.org/article/10.3389/fpls.2016.01836

65. Johnston PA, Niks RE, Meiyalaghan V, Blanchet E, Pickering R. Rph22: mapping of a novel leaf rust resistance gene introgressed from the non-host Hordeum bulbosum L. into cultivated barley (Hordeum vulgare L.). Theor Appl Genet. 2013;126:1613-25. Available from: http://dx.doi.org/10.1007/s00122-013-2078-9

66. Risk JM, Selter LL, Chauhan H, Krattinger SG, Kumlehn J, Hensel G, et al. The wheat Lr34 gene provides resistance against multiple fungal pathogens in barley. Plant Biotechnol J. 2013;11:847-54. Available from: http://dx.doi.org/10.1111/pbi.12077

67. Krattinger SG, Sucher J, Selter LL, Chauhan H, Zhou B, Tang M, et al. The wheat durable, multipathogen resistance gene Lr34 confers partial blast resistance in rice. Plant Biotechnol J. 2016;14:1261-8. Available from: http:// dx.doi.org/10.1111/pbi.12491

68. Tanaka M, Nakashiki H, Tosa Y, Nakayashiki H, Tosa Y. Population structure of Eleusine isolates of Pyricularia oryzae and its evolutionary implications. J Gen Plant Pathol. 2009;75:173-80.

69. Delventhal R, Falter C, Strugala R, Zellerhoff N, Schaffrath U. Ectoparasitic growth of Magnaporthe on barley triggers expression of the putative barley wax biosynthesis gene CYP96B22 which is involved in penetration resistance. BMC Plant Biol. 2014;14:26. Available from: http://www. biomedcentral.com/1471-2229/14/26

70. Atienza S, Jafary H, Niks R. Accumulation of genes for susceptibility to rust fungi for which barley is nearly a nonhost results in two barley lines with extreme multiple susceptibility. Planta. 2004;220:71-9. Available from: http:// dx.doi.org/10.1007/s00425-004-1319-1

71. Carver TLW, Ingerson-Morris SM. Effects of inoculum density on germling development by Erysiphe graminis f. sp. avenae in relation to induced resistance of oat cells to appressorial penetration. Mycol Res. 1989;92:18-24. Available from: http://www.sciencedirect.com/science/article/pii/ S0953756289800905

72. Hoogkamp TJ, Chen WQ, Niks RE. Specificity of prehaustorial resistance to Puccinia hordei and to two inappropriate rust fungi in barley. Phytopathology. 1998;88:856-61. Available from: http://dx.doi.org/10.1094/ PHYTO.1998.88.8.856

73. Chen X, Hedley PE, Morris J, Liu H, Niks RE, Waugh R. Combining genetica genomics and bulked segregant analysis-based differential expression: an approach to gene localization. Theor Appl Genet. 2011;122:1375-83. Available from: http://dx.doi.org/10.1007/s00122-011-1538-3

74. Liu H, McNicol J, Bayer M, Morris JA, Cardle L, Marshall DF, et al. Highly parallel gene-to-BAC addressing using microarrays. BioTechniques. 2011;50: 165-72. Available from: www.BioTechniques.com/article/113627

75. Mayer KFX, Martis M, Hedley PE, Simková H, Liu H, Morris JA, et al. Unlocking the barley genome by chromosomal and comparative genomics. Plant Cell. 2011;23:1249-63. Available from: http://dx.doi.org/10.1105/tpc.110.082537

76. Brazma A, Parkinson H, Sarkans U, Shojatalab M, Vilo J, Abeygunawardena N, et al. ArrayExpress - a public repository for microarray gene expression data at the EBI. Nucleic Acids Res. 2003;31:68-71. Available from: http://www. ncbi.nlm.nih.gov/pmc/articles/PMC165538/

77. Kolesnikov N, Hastings E, Keays M, Melnichuk O, Tang YA, Williams E, et al. ArrayExpress update-simplifying data submissions. Nucleic Acids Res. 2015; 43:D1113-6. Available from: http://europepmc.org/abstract/MED/25361974

78. Quackenbush J, Cho J, Lee D, Liang F, Holt I, Karamycheva S, et al. The TIGR gene indices: analysis of gene transcript sequences in highly sampled eukaryotic species. Nucleic Acids Res. 2001;29:159-64. Available from: http:// nar.oxfordjournals.org/content/29/1/159.abstract

79. TGI database. Available from: http://compbio.dfci.harvard.edu/tgi/. Accessed 08 May 2017.

80. HarvEST, EST database-viewing software. Available from: http://harvest.ucr. edu/. Accessed 27 Aug 2012

81. Altschul SF, Gish W, Miller W, Myers EW, Lipman DJ. Basic local alignment search tool. J Mol Biol. 1990;215:403-10. Available from: http://dx.doi.org/10. 1016/S0022-2836(05)80360-2

82. Altschul SF, Madden TL, Schäffer AA, Zhang J, Zhang Z, Miller W, et al. Gapped BLAST and PSI-BLAST: a new generation of protein database search programs. Nucleic Acids Res. 1997;25:3389-402. 
83. NCBI Resource Coordinators. Database resources of the National Center for biotechnology information. Nucleic Acids Res. 2017:45:D12-7. Oxford University Press. Available from: http://www.ncbinlm.nih.gov/pmc/articles/ PMC5210554/

84. Conesa A, Götz S, García-Gómez JM, Terol J, Talón M, Robles M. Blast2GO: a universal tool for annotation, visualization and analysis in functional genomics research. Bioinformatics. 2005;21:3674-6.

85. International Barley Genome Sequencing Consortium IBSC, Mayer KF, Waugh R, Brown JW, Schulman A, Langridge P, et al. A physical, genetic and functional sequence assembly of the barley genome. Nature. 2012;491: 711-6. Available from: http://www.ncbi.n/m.nih.gov/pubmed/23075845

86. Lohse M, Nagel A, Herter T, May P, Schroda M, Zrenner R, et al. Mercator: a fast and simple web server for genome scale functional annotation of plant sequence data. Plant Cell Environ. 2014;37:1250-8. Available from: http://dx. doi.org/10.1111/pce.12231

87. Bolger ME, Arsova B, Usadel B. Plant genome and transcriptome annotations: from misconceptions to simple solutions. Brief Bioinform. 2017: bbw135. Available from: https://academic.oup.com/bib/article-lookup/doi/ 10.1093/bib/bbw135

88. Sturn A, Quackenbush J, Trajanoski Z. Genesis: cluster analysis of microarray data. Bioinformatics. 2002;18:207-8. Available from: http://bioinformatics. oxfordjournals.org/content/18/1/207.abstract

\section{Submit your next manuscript to BioMed Central} and we will help you at every step:

- We accept pre-submission inquiries

- Our selector tool helps you to find the most relevant journal

- We provide round the clock customer support

- Convenient online submission

- Thorough peer review

- Inclusion in PubMed and all major indexing services

- Maximum visibility for your research

Submit your manuscript at www.biomedcentral.com/submit 